TRANSACTIONS OF THE

AMERICAN MATHEMATICAL SOCIETY

Volume 361, Number 12, December 2009, Pages 6447-6473

S 0002-9947(09)04860-0

Article electronically published on July 14, 2009

\title{
ALPHA-DETERMINANT CYCLIC MODULES AND JACOBI POLYNOMIALS
}

\author{
KAZUFUMI KIMOTO, SHO MATSUMOTO, AND MASATO WAKAYAMA, \\ WITH AN APPENDIX BY KAZUFUMI KIMOTO
}

Dedicated to Professor Masaaki Yoshida on his sixtieth birthday.

\begin{abstract}
For positive integers $n$ and $l$, we study the cyclic $\mathcal{U}\left(\mathfrak{g l}_{n}\right)$-module generated by the $l$-th power of the $\alpha$-determinant $\operatorname{det}^{(\alpha)}(X)$. This cyclic module is isomorphic to the $n$-th tensor space $\mathcal{S}^{l}\left(\mathbb{C}^{n}\right)^{\otimes n}$ of the symmetric $l$-th tensor space of $\mathbb{C}^{n}$ for all but finitely many exceptional values of $\alpha$. If $\alpha$ is exceptional, then the cyclic module is equivalent to a proper submodule of $\mathcal{S}^{l}\left(\mathbb{C}^{n}\right)^{\otimes n}$, i.e. the multiplicities of several irreducible subrepresentations in the cyclic module are smaller than those in $\mathcal{S}^{l}\left(\mathbb{C}^{n}\right)^{\otimes n}$. The degeneration of each isotypic component of the cyclic module is described by a matrix whose size is given by a Kostka number and whose entries are polynomials in $\alpha$ with rational coefficients. In particular, we determine the matrix completely when $n=2$. In this case, the matrix becomes a scalar and is essentially given by a classical Jacobi polynomial. Moreover, we prove that these polynomials are unitary.

In the Appendix, we consider a variation of the spherical Fourier transformation for $\left(\mathfrak{S}_{n l}, \mathfrak{S}_{l}^{n}\right)$ as a main tool for analyzing the same problems, and describe the case where $n=2$ by using the zonal spherical functions of the Gelfand pair $\left(\mathfrak{S}_{2 l}, \mathfrak{S}_{l}^{2}\right)$.
\end{abstract}

\section{INTRODUCTION}

Let $\mathcal{A}\left(\mathrm{Mat}_{n}\right)$ be the associative $\mathbb{C}$-algebra consisting of polynomials in variables $\left\{x_{i j}\right\}_{1 \leq i, j \leq n}$. We introduce a $\mathcal{U}\left(\mathfrak{g l}_{n}\right)$-module structure on $\mathcal{A}\left(\mathrm{Mat}_{n}\right)$, where $\mathcal{U}\left(\mathfrak{g l}_{n}\right)$ is the universal enveloping algebra of the general linear Lie algebra $\mathfrak{g l}_{n}=\mathfrak{g l}_{n}(\mathbb{C})$, by defining

$$
\rho_{\mathfrak{g r}_{n}}\left(E_{i j}\right) f=\sum_{k=1}^{n} x_{i k} \frac{\partial f}{\partial x_{j k}} \quad\left(f \in \mathcal{A}\left(\mathrm{Mat}_{n}\right)\right),
$$

which is obtained as a differential representation of the translation of $G L_{n}=$ $G L_{n}(\mathbb{C})$.

Received by the editors October 29, 2007.

2000 Mathematics Subject Classification. Primary 22E47, 33C45; Secondary 43A90, 13A50.

Key words and phrases. Alpha-determinant, cyclic modules, Jacobi polynomials, singly confluent Heun ODE, permanent, Kostka numbers, irreducible decomposition, spherical Fourier transformation, zonal spherical functions, Gelfand pair.

The second author was partially supported by Grant-in-Aid for JSPS Fellows No. 17006193.

The third author was partially supported by Grant-in-Aid for Exploratory Research No. 18654005 .

(C)2009 American Mathematical Society 
Since the determinant $\operatorname{det}(X)$ of the matrix $X=\left(x_{i j}\right)_{1 \leq i, j \leq n}$ is a relative $G L_{n^{-}}$ invariant in $\mathcal{A}\left(\mathrm{Mat}_{n}\right)$, obviously the linear span $\mathbb{C} \cdot \operatorname{det}(X)$ is a one-dimensional irreducible (highest weight) $\mathcal{U}\left(\mathfrak{g l}_{n}\right)$-submodule of $\mathcal{A}\left(\mathrm{Mat}_{n}\right)$. This submodule is equivalent to the skew-symmetric tensor representation $\bigwedge^{n}\left(\mathbb{C}^{n}\right)$ of the natural representation of $\mathcal{U}\left(\mathfrak{g l}_{n}\right)$ on $\mathbb{C}^{n}$. The symmetric counterpart of the determinant is the permanent $\operatorname{per}(X)$ given by

$$
\operatorname{per}(X)=\sum_{\sigma \in \mathfrak{S}_{n}} x_{\sigma(1) 1} x_{\sigma(2) 2} \cdots x_{\sigma(n) n} .
$$

Although $\operatorname{per}(X)$ is not a relative invariant of $G L_{n}$, the cyclic module $\rho_{\mathfrak{g l}_{n}}\left(\mathcal{U}\left(\mathfrak{g l}_{n}\right)\right)$. $\operatorname{per}(X)$ (i.e. the smallest invariant subspace in $\mathcal{A}\left(\mathrm{Mat}_{n}\right)$ containing $\operatorname{per}(X)$ ) is irreducible and equivalent to the symmetric tensor representation $\mathcal{S}^{n}\left(\mathbb{C}^{n}\right)$ of the natural representation.

The $\alpha$-determinant of $X$ is defined by

$$
\operatorname{det}^{(\alpha)}(X)=\sum_{\sigma \in \mathfrak{S}_{n}} \alpha^{\nu(\sigma)} x_{\sigma(1) 1} x_{\sigma(2) 2} \cdots x_{\sigma(n) n},
$$

where $\nu(\sigma)$ is $n$ minus the number of cycles in $\sigma \in \mathfrak{S}_{n}$. The notion of the $\alpha$ determinant was first introduced in [16] in order to describe the coefficients in the expansion of $\operatorname{det}(I-\alpha A)^{-1 / \alpha}$, which is used to treat the multivariate binomial and negative binomial distributions in a unified way. Later, it was also used to define a certain random process in 13. We note that a pfaffian analogue ( $\alpha$-pfaffian) was introduced and studied from the same (probability theoretic) viewpoint by the second author in [11.

The $\alpha$-determinant is a common generalization of (and/or an interpolation between) the determinant and permanent since $\operatorname{det}^{(-1)}(X)=\operatorname{det}(X)$ and $\operatorname{det}^{(1)}(X)=$ $\operatorname{per}(X)$. In this sense, the $\alpha$-determinant cyclic module $\rho_{\mathfrak{g l}_{n}}\left(\mathcal{U}\left(\mathfrak{g l}_{n}\right)\right) \cdot \operatorname{det}^{(\alpha)}(X)$ is regarded as an interpolation between two irreducible representations - the skewsymmetric tensor representation and the symmetric tensor representation. In [12, the second and third authors determined the structure of the $\mathcal{U}\left(\mathfrak{g l}_{n}\right)$-cyclic module $\rho_{\mathfrak{g l}_{n}}\left(\mathcal{U}\left(\mathfrak{g l}_{n}\right)\right) \cdot \operatorname{det}^{(\alpha)}(X)$. The irreducible decomposition of $\rho_{\mathfrak{g l}_{n}}\left(\mathcal{U}\left(\mathfrak{g l}_{n}\right)\right) \cdot \operatorname{det}^{(\alpha)}(X)$ is given by

$$
\rho_{\mathfrak{g l}_{n}}\left(\mathcal{U}\left(\mathfrak{g l}_{n}\right)\right) \cdot \operatorname{det}^{(\alpha)}(X) \cong \bigoplus_{\substack{\lambda \vdash n \\ f_{\lambda}(\alpha) \neq 0}}\left(\mathcal{M}_{n}^{\lambda}\right)^{\oplus f^{\lambda}} .
$$

Here $\mathcal{M}_{n}^{\lambda}$ denotes the irreducible highest weight $\mathcal{U}\left(\mathfrak{g l}_{n}\right)$-module of highest weight $\lambda$ (we identify the highest weight and the corresponding partition), $f^{\lambda}$ the number of standard tableaux with shape $\lambda$ and $f_{\lambda}(\alpha)$ the (modified) content polynomial [10] for $\lambda$ defined by

$$
f_{\lambda}(\alpha)=\prod_{i=1}^{\ell(\lambda)} \prod_{j=1}^{\lambda_{i}}(1+(j-i) \alpha) .
$$

In other words, the structure of $\rho_{\mathfrak{g l}_{n}}\left(\mathcal{U}\left(\mathfrak{g l}_{n}\right)\right) \cdot \operatorname{det}^{(\alpha)}(X)$ changes drastically when $\alpha= \pm 1 / k(k=1,2, \ldots, n-1)$. This result implies that $\operatorname{det}^{(\alpha)}(X)$ may exhibit some special feature like $\operatorname{det}(X)$ and/or $\operatorname{per}(X)$ for such special values of $\alpha$. Actually, when $\alpha=-1 / k$ for some $k$, $\operatorname{det}^{(-1 / k)}(X)$ has a property analogous to the alternating property of the determinant. Based on this fact, for instance, we can 
construct a relative $G L_{n}$-invariant from $\operatorname{det}^{(-1 / k)}(X)$ (see [8]). It is worth noting that we also introduced an object analogous to the $\alpha$-determinant $\operatorname{det}_{q}^{(\alpha)}(X)$ in the quantum matrix algebra and studied the quantum enveloping algebra cyclic module $\mathcal{U}_{q}\left(\mathfrak{g l}_{n}\right) \cdot \operatorname{det}_{q}^{(\alpha)}(X)$ in [9]. Compared to the classical case [12], the cyclic module in the quantum case is much more complicated and has a rich structure.

As a next stage, as in the beginning of the study of infinite dimensional representation theory by Gel'fand and Nalmark [5] in the middle of the last century, it is natural to proceed with the study of the cyclic modules $\rho_{\mathfrak{g r}_{n}}\left(\mathcal{U}\left(\mathfrak{g l}_{n}\right)\right) \cdot \operatorname{det}^{(\alpha)}(X)^{s}$ for $s \in \mathbb{C}$ under a suitable reformulation (see Section 5.2). In this case, the cyclic modules $\rho_{\mathfrak{g l}_{n}}\left(\mathcal{U}\left(\mathfrak{g l}_{n}\right)\right) \cdot \operatorname{det}^{(\alpha)}(X)^{s}$ are not finite dimensional in general. Actually, if $s$ is not a non-negative integer, then $\operatorname{det}^{(\alpha)}(X)^{s}$ is no longer a polynomial and $\rho_{\mathfrak{g l}_{n}}\left(\mathcal{U}\left(\mathfrak{g l}_{n}\right)\right) \cdot \operatorname{det}^{(\alpha)}(X)^{s}$ becomes infinite dimensional unless $\alpha=-1$. In contrast, when $s=l$ is a positive integer, $\rho_{\mathfrak{g l}_{n}}\left(\mathcal{U}\left(\mathfrak{g l}_{n}\right)\right) \cdot \operatorname{det}^{(\alpha)}(X)^{l}$ is a submodule of the polynomial algebra $\mathcal{A}\left(\mathrm{Mat}_{n}\right)$ and is finite dimensional.

In this article, we treat the finite-dimensional cases, that is, we study the cyclic module $\rho_{\mathfrak{g l}_{n}}\left(\mathcal{U}\left(\mathfrak{g l}_{n}\right)\right) \cdot \operatorname{det}^{(\alpha)}(X)^{l}$ for a given positive integer $l$. We first show that the irreducible decomposition is given in the form

$$
\rho_{\mathfrak{g r}_{n}}\left(\mathcal{U}\left(\mathfrak{g l}_{n}\right)\right) \cdot \operatorname{det}^{(\alpha)}(X)^{l} \cong \bigoplus_{\lambda \vdash n l}\left(\mathcal{M}_{n}^{\lambda}\right)^{\oplus m_{n, l}^{\lambda}(\alpha)},
$$

where $m_{n, l}^{\lambda}(\alpha)$ denotes the multiplicity of the irreducible submodule with highest weight $\lambda$ which satisfies $0 \leq m_{n, l}^{\lambda}(\alpha) \leq K_{\lambda\left(l^{n}\right)}$ (Theorem [3.1). Here $K_{\lambda \mu}$ is the Kostka number, defined as the number of semi-standard tableaux of shape $\lambda$ and weight $\mu$. Moreover, there exists a certain matrix $F_{n, l}^{\lambda}(\alpha)$ of size $K_{\lambda\left(l^{n}\right)}$, called the transition matrix for $\lambda$, whose entries are polynomials in $\alpha$ such that $m_{n, l}^{\lambda}(\alpha)=$ $\operatorname{rk} F_{n, l}^{\lambda}(\alpha)$ for each $\lambda$. By this fact, for all but finitely many $\alpha$, we have $m_{n, l}^{\lambda}(\alpha)=$ $K_{\lambda\left(l^{n}\right)}$ for any $\lambda$. In other words, the cyclic module $\rho_{\mathfrak{g l}_{n}}\left(\mathcal{U}\left(\mathfrak{g l}_{n}\right)\right) \cdot \operatorname{det}^{(\alpha)}(X)^{l}$ is equivalent to the space $\mathcal{S}^{l}\left(\mathbb{C}^{n}\right)^{\otimes n}$ of symmetric $l$-tensors on $\mathbb{C}^{n}$ for almost all $\alpha$. We note that $F_{n, 1}^{\lambda}(\alpha)$ is a scalar matrix $f^{\lambda}(\alpha) \cdot I$ (see (1.2) and (1.3)).

Consequently, we have to describe the transition matrix $F_{n, l}^{\lambda}(\alpha)$ and/or its rank $\operatorname{rk} F_{n, l}^{\lambda}(\alpha)\left(=m_{n, l}^{\lambda}(\alpha)\right)$ explicitly. When $n=2$, we can completely determine the explicit form of the transition matrices (see Section 4). In this case, each transition matrix is a scalar and is given by a classical Jacobi polynomial. (To be precise, the scalar satisfies a singly confluent Heun ordinary differential equation with respect to $\alpha$. See Corollary [4.2.) In other words, the Jacobi polynomials play the role of the content polynomials. Moreover, one can show that these Jacobi polynomials are unitary, and hence that the multiplicity $m_{2, l}^{\lambda}(\alpha)$ is non-zero unless $|\alpha|=1$ for each partition $\lambda$ of $2 l$. These are our main results.

Here we should remark that the Jacobi polynomial does not appear as a spherical function (i.e. a matrix coefficient of a representation) in our story, hence it is important to clarify the reason why the transition matrix becomes a (unitary) Jacobi polynomial when $n=2$. It seems a far-off goal at present to describe the transition matrices when $n \geq 3$. In fact, we can give explicit expressions for transition matrices only in a few special cases. It is not clear whether (the entries of) the transition matrices are given by certain special polynomials. We leave these problems for future study. 
This paper is organized as follows. In Section 2, we recall the $G L_{n}$-module structure of the tensor space $\mathcal{S}^{l}\left(\mathbb{C}^{n}\right)^{\otimes n}$. This space is the basic one for the study of $\alpha$-determinant cyclic modules. In Section 3, we study the structure of the cyclic module $\mathcal{U}\left(\mathfrak{g l}_{n}\right) \cdot \operatorname{det}^{(\alpha)}(X)^{l}$. The transition matrix, which determines the multiplicity of the irreducible component in the cyclic module, is defined in this section. In Section 4, we exclusively deal with the simple case where $n=2$. As stated above, the transition matrix in this case is explicitly given by a classical Jacobi polynomial. In Section 5, we state a conjecture concerning the permanent cyclic module $\mathcal{U}\left(\mathfrak{g l}_{n}\right)$. $\operatorname{per}(X)^{l}(\alpha=1$ case), introduce a certain suitable reformulation of our problem for the general complex power cases (i.e. $\mathcal{U}\left(\mathfrak{g l}_{n}\right) \cdot \operatorname{det}^{(\alpha)}(X)^{s}$ for $s \in \mathbb{C}$ ) and give a remark on $\varphi$-immanant cyclic modules which provide a generalization of the situation.

In the Appendix, we investigate our problems by another approach. We adopt a variation of the spherical Fourier transformation for $\left(\mathfrak{S}_{n l}, \mathfrak{S}_{l}^{n}\right)$ as a main tool by which to analyze the structure of $\mathcal{U}\left(\mathfrak{g l}_{n}\right) \cdot \operatorname{det}^{(\alpha)}(X)^{l}$, and give another proof of the results in Section 3. We also describe the transition matrices in the case where $n=2$ by using the zonal spherical functions of the Gelfand pair $\left(\mathfrak{S}_{2 l}, \mathfrak{S}_{l}^{2}\right)$.

\section{Preliminary Results on the Representation of $\mathcal{U}\left(\mathfrak{g l}_{n}\right)$}

Let $\mathbb{Z}_{+}$be the set of all non-negative integers. For a positive integer $n$, we put $[n]=\{1,2, \ldots, n\}$. Let $\boldsymbol{e}_{1}, \ldots, \boldsymbol{e}_{n}$ be the standard basis of $\mathbb{C}^{n}$. The symmetric $l$-tensor space $\mathcal{S}^{l}\left(\mathbb{C}^{n}\right)$ is the set of all polynomials of degree $l$ in variables $\boldsymbol{e}_{i}$ and is expressed as follows:

$$
\mathcal{S}^{l}\left(\mathbb{C}^{n}\right)=\bigoplus_{\substack{m_{1}, \ldots, m_{n} \in \mathbb{Z}_{+} \\ m_{1}+\cdots+m_{n}=l}} \mathbb{C} \cdot \boldsymbol{e}_{1}^{m_{1}} \boldsymbol{e}_{2}^{m_{2}} \cdots \boldsymbol{e}_{n}^{m_{n}}
$$

Let $\mathbb{M}_{n, l}$ be the set of all $\mathbb{Z}_{+}$-matrices of size $n$ such that the sum of entries in each column is equal to $l$ :

$$
\mathbb{M}_{n, l}=\left\{M=\left(m_{i j}\right)_{1 \leq i, j \leq n} \mid m_{i j} \in \mathbb{Z}_{+}, \quad \sum_{i=1}^{n} m_{i j}=l \quad(1 \leq j \leq n)\right\} .
$$

Put

$$
\boldsymbol{e}^{M}=\boldsymbol{e}_{1}^{m_{11}} \boldsymbol{e}_{2}^{m_{21}} \cdots \boldsymbol{e}_{n}^{m_{n 1}} \otimes \cdots \otimes \boldsymbol{e}_{1}^{m_{1 n}} \boldsymbol{e}_{2}^{m_{2 n}} \cdots \boldsymbol{e}_{n}^{m_{n n}}
$$

for each $M \in \mathbb{M}_{n, l}$. Then the tensor space $\mathcal{S}^{l}\left(\mathbb{C}^{n}\right)^{\otimes n}$ is given by

$$
\mathcal{S}^{l}\left(\mathbb{C}^{n}\right)^{\otimes n}=\bigoplus_{M \in \mathbb{M}_{n, l}} \mathbb{C} \cdot \boldsymbol{e}^{M}
$$

The universal enveloping algebra $\mathcal{U}\left(\mathfrak{g l}_{n}\right)$ acts on $\mathbb{C}^{n}$ in a natural way: $E_{i j} \cdot \boldsymbol{e}_{k}=$ $\delta_{j k} \boldsymbol{e}_{i}$, where $\delta_{j k}$ is Kronecker's delta. This action induces the action of $\mathcal{U}\left(\mathfrak{g l}_{n}\right)$ on $\mathcal{S}^{l}\left(\mathbb{C}^{n}\right)^{\otimes n}$ as

$$
E_{p q} \cdot e^{M}=\sum_{k=1}^{n} m_{q k} e^{M+R_{k}^{p q}} \quad\left(1 \leq p, q \leq n, M=\left(m_{i j}\right)_{1 \leq i, j \leq n} \in \mathbb{M}_{n, l}\right),
$$

where $R_{k}^{p q}$ is the matrix of size $n$ whose $(i, j)$-entry is equal to $\left(\delta_{i p}-\delta_{i q}\right) \delta_{j k}$. We note that $R_{k}^{p q}=-R_{k}^{q p}$. The irreducible decomposition of the $\mathcal{U}\left(\mathfrak{g l}_{n}\right)$-module $\mathcal{S}^{l}\left(\mathbb{C}^{n}\right)^{\otimes n}$ 
is well known and given by

$$
\mathcal{S}^{l}\left(\mathbb{C}^{n}\right)^{\otimes n} \cong \bigoplus_{\lambda \vdash n l}\left(\mathcal{M}_{n}^{\lambda}\right)^{\oplus K_{\lambda\left(l^{n}\right)}} ;
$$

see e.g. [4, 17]. Here $\mathcal{M}_{n}^{\lambda}$ denotes the highest weight module of $\mathcal{U}\left(\mathfrak{g l}_{n}\right)$ with highest weight $\lambda=\left(\lambda_{1}, \ldots, \lambda_{n}\right)$ and $K_{\lambda\left(l^{n}\right)}$ denotes the Kostka number which is defined as the number of semi-standard tableaux of shape $\lambda$ and weight $\left(l^{n}\right)=(l, l, \ldots, l)$.

Example 2.1. Let $n=2$. Then $\mathbb{M}_{2, l}=\left\{\left(\begin{array}{cc}r & s \\ l-r & l-s\end{array}\right) \mid 0 \leq r, s \leq l\right\}$. When $l=2$, we see that

$$
\begin{aligned}
& E_{21} \cdot e^{\left(\begin{array}{ll}
2 & 1 \\
0 & 1
\end{array}\right)}=2 e^{\left(\begin{array}{ll}
2 & 1 \\
0 & 1
\end{array}\right)+\left(\begin{array}{cc}
-1 & 0 \\
1 & 0
\end{array}\right)}+e^{\left(\begin{array}{ll}
2 & 1 \\
0 & 1
\end{array}\right)+\left(\begin{array}{cc}
0 & -1 \\
0 & 1
\end{array}\right)} \\
& =2 \boldsymbol{e}^{\left(\begin{array}{ll}
1 & 1 \\
1 & 1
\end{array}\right)}+\boldsymbol{e}^{\left(\begin{array}{ll}
2 & 0 \\
0 & 2
\end{array}\right)}=2 \boldsymbol{e}_{1} \boldsymbol{e}_{2} \otimes \boldsymbol{e}_{1} \boldsymbol{e}_{2}+\boldsymbol{e}_{1}^{2} \otimes \boldsymbol{e}_{2}^{2}, \\
& E_{11} \cdot e^{\left(\begin{array}{ll}
2 & 1 \\
0 & 1
\end{array}\right)}=3 \boldsymbol{e}^{\left(\begin{array}{ll}
2 & 1 \\
0 & 1
\end{array}\right)}=3 \boldsymbol{e}_{1}^{2} \otimes \boldsymbol{e}_{1} \boldsymbol{e}_{2}
\end{aligned}
$$

for instance. The irreducible decomposition of $\mathcal{S}^{l}\left(\mathbb{C}^{2}\right)^{\otimes 2}$ is given as

$$
\mathcal{S}^{l}\left(\mathbb{C}^{2}\right)^{\otimes 2} \cong \bigoplus_{s=0}^{l} \mathcal{M}_{2}^{(2 l-s, s)} .
$$

The following lemma plays a fundamental role in the discussion below.

Lemma 2.2. Let $I_{n}$ be the identity matrix of size $n$. Then $\mathcal{S}^{l}\left(\mathbb{C}^{n}\right)^{\otimes n}=\mathcal{U}\left(\mathfrak{g l}_{n}\right) \cdot e^{l I_{n}}$, that is, the vector $\boldsymbol{e}^{l I_{n}}=\boldsymbol{e}_{1}^{l} \otimes \boldsymbol{e}_{2}^{l} \otimes \cdots \otimes \boldsymbol{e}_{n}^{l}$ is a cyclic vector of the $\mathcal{U}\left(\mathfrak{g l}_{n}\right)$-module $\mathcal{S}^{l}\left(\mathbb{C}^{n}\right)^{\otimes n}$.

Proof. Fix a positive integer $l$. Let $\widetilde{\mathbb{M}}_{n, l}$ be the subset

$$
\widetilde{\mathbb{M}}_{n, l}=\left\{M \in \mathbb{M}_{n, l} \mid e^{M} \in \mathcal{U}\left(\mathfrak{g l}_{n}\right) \cdot e^{l I_{n}}\right\}
$$

of $\mathbb{M}_{n, l}$. Let us prove that $\mathcal{S}^{l}\left(\mathbb{C}^{n}\right)^{\otimes n}=\bigoplus_{M \in \widetilde{\mathbb{M}}_{n, l}} \mathbb{C} \cdot e^{M}$, or equivalently that

$$
\widetilde{\mathbb{M}}_{n, l} \supset \mathbb{M}_{n, l}
$$

by induction on $n$.

The universal enveloping algebra $\mathcal{U}\left(\mathfrak{g l}_{n-1}\right)$ is embedded in $\mathcal{U}\left(\mathfrak{g l}_{n}\right)$ as a subalgebra in a natural way. Assume that the inclusion (2.2) holds up to $n-1$. Then the matrices of the form

$$
M^{\prime} \oplus(l)=\left(\begin{array}{ccc|c} 
& & 0 \\
& M^{\prime} & & \vdots \\
& & & 0 \\
\hline 0 & \ldots & 0 & l
\end{array}\right) \quad\left(M^{\prime} \in \mathbb{M}_{n-1, l}\right)
$$

are contained in $\widetilde{\mathbb{M}}_{n, l}$ by the induction hypothesis. Applying several $E_{j n}$ 's successively to $e^{M^{\prime} \oplus(l)}$ suitably many times, we first see that any matrix of the form

$$
\left(\begin{array}{ccc|c} 
& & m_{1 n} \\
& M^{\prime} & & \vdots \\
& & & m_{n-1, n} \\
\hline 0 & \ldots & 0 & m_{n n}
\end{array}\right) \quad\left(M^{\prime} \in \mathbb{M}_{n-1, l}, \sum_{i=1}^{n} m_{i n}=l\right)
$$

belongs to $\widetilde{\mathbb{M}}_{n, l}$. 
Next, we put

$$
\begin{array}{r}
\mathbb{M}_{n, l}(p)=\left\{\left(m_{i j}\right)_{1 \leq i, j \leq n} \in \mathbb{M}_{n, l} \mid m_{i j}=0 \text { if } j \in\{p+1, p+2, \ldots, n-1\}\right. \\
\text { and } i \in\{1,2, \ldots, j-1, n\}\}
\end{array}
$$

for each $0 \leq p \leq n-1$. Notice that $\mathbb{M}_{n, l}(0) \subset \mathbb{M}_{n, l}(1) \subset \cdots \subset \mathbb{M}_{n, l}(n-1)=\mathbb{M}_{n, l}$. We show that $\mathbb{M}_{n, l}(p) \subset \widetilde{\mathbb{M}}_{n, l}$ for any $0 \leq p \leq n-1$ by induction on $p$. By definition, we see that any element in $\mathbb{M}_{n, l}(0)$ is of the form (2.3), so we have $\mathbb{M}_{n, l}(0) \subset \widetilde{\mathbb{M}}_{n, l}$. Assume $\mathbb{M}_{n, l}(p-1) \subset \widetilde{\mathbb{M}}_{n, l}$ for $1 \leq p \leq n-1$. Take any matrix $M=\left(m_{i j}\right)_{1 \leq i, j \leq n}$ in $\mathbb{M}_{n, l}(p)$, and put $\widetilde{M}=M+\sum_{i=1}^{p-1} m_{i p} R_{p}^{p i}+m_{n p} R_{p}^{p n}$. Equivalently, $\widetilde{M}$ is a matrix defined by

$$
\widetilde{m}_{i p}= \begin{cases}m_{1 p}+m_{2 p}+\cdots+m_{p-1, p}+m_{p, p}+m_{n, p} & \text { if } i=p, \\ m_{i p} & \text { if } p+1 \leq i \leq n-1, \\ 0 & \text { otherwise, }\end{cases}
$$

and $\widetilde{m}_{i j}=m_{i j}$ for $j \neq p$. It is easy to see that $\widetilde{M} \in \mathbb{M}_{n, l}(p-1)$. Then, using Lemma 2.3 below, we get

$$
\begin{aligned}
E_{1 p}^{m_{1 p}} E_{2 p}^{m_{2 p}} \cdots E_{p-1, p}^{m_{p-1, p}} E_{n p}^{m_{n p}} \cdot e^{\widetilde{M}} & \\
& \equiv(\text { non-zero constant }) \times e^{M} \quad\left(\bmod \bigoplus_{N \in \mathbb{M}_{n, l}(p-1)} \mathbb{C} \cdot e^{N}\right) .
\end{aligned}
$$

Therefore we have $M \in \widetilde{\mathbb{M}}_{n, l}$ by the induction hypothesis on $p$, and hence $\mathbb{M}_{n, l}(p) \subset$ $\widetilde{\mathbb{M}}_{n, l}$. In particular, we get $\mathbb{M}_{n, l}=\mathbb{M}_{n, l}(n-1) \subset \widetilde{\mathbb{M}}_{n, l}$, which is the desired conclusion.

In the proof, we have used the following lemma which is readily verified.

Lemma 2.3. Suppose that $M \in \mathbb{M}_{n, l}(p-1)$ and $1 \leq i, k \leq p-1$. Then $M+R_{k}^{i p}=$ $M-R_{k}^{p i} \in \mathbb{M}_{n, l}(p-1)$. In particular,

$$
E_{i p}^{d} \boldsymbol{e}^{M} \equiv m_{p p}\left(m_{p p}-1\right) \cdots\left(m_{p p}-d+1\right) \boldsymbol{e}^{M-d R_{p}^{p i}} \quad\left(\bmod \bigoplus_{N \in \mathbb{M}_{n, l}(p-1)} \mathbb{C} \cdot \boldsymbol{e}^{N}\right)
$$

holds for $d \geq 1$.

The linear map $\rho_{\mathfrak{g l}_{n}}: \mathcal{U}\left(\mathfrak{g l}_{n}\right) \rightarrow \operatorname{End}_{\mathbb{C}}\left(\mathcal{A}\left(\mathrm{Mat}_{n}\right)\right)$ defined by

$$
\left(\rho_{\mathfrak{g l}_{n}}\left(E_{i j}\right) \cdot f\right)(X)=\sum_{k=1}^{n} x_{i k} \frac{\partial f}{\partial x_{j k}}(X) \quad\left(1 \leq i, j \leq n, \quad f \in \mathcal{A}\left(\mathrm{Mat}_{n}\right)\right)
$$

determines a representation of $\mathcal{U}\left(\mathfrak{g l}_{n}\right)$ on $\mathcal{A}\left(\mathrm{Mat}_{n}\right)$. We abbreviate $\rho_{\mathfrak{g l}_{n}}\left(E_{i j}\right)$ as $E_{i j}$.

For each $M \in \mathbb{M}_{n, l}$, put $X^{M}=\prod_{i, j=1}^{n} x_{i j}^{m_{i j}}$. The action of $\mathcal{U}\left(\mathfrak{g l}_{n}\right)$ to $X^{M}$ is given by

$$
E_{p q} \cdot X^{M}=\sum_{k=1}^{n} m_{q k} X^{M+R_{k}^{p q}} \quad(1 \leq p, q \leq n)
$$


Combining this with (2.1) and Lemma 2.2, we see that the linear map $e^{M} \mapsto X^{M}$ $\left(M \in \mathbb{M}_{n, l}\right)$ gives the isomorphism

$$
\mathcal{S}^{l}\left(\mathbb{C}^{n}\right)^{\otimes n} \cong \bigoplus_{M \in \mathbb{M}_{n, l}} \mathbb{C} \cdot X^{M}=\mathcal{U}\left(\mathfrak{g l}_{n}\right) \cdot x_{11}^{l} x_{22}^{l} \cdots x_{n n}^{l} \subset \mathcal{A}\left(\mathrm{Mat}_{n}\right)
$$

\section{The CYCLIC MOdules $\mathcal{U}\left(\mathfrak{g l}_{n}\right) \cdot \operatorname{det}^{(\alpha)}(X)^{l}$}

3.1. $\alpha$-determinants and intertwiners. Let $\alpha$ be a complex number. We consider the cyclic module $\mathcal{U}\left(\mathfrak{g l}_{n}\right) \cdot \operatorname{det}^{(\alpha)}(X)^{l}$ for a positive integer $l$.

When $\alpha=0$, we have $\operatorname{det}^{(0)}(X)=x_{11} x_{22} \cdots x_{n n}$. From (2.4) we obtain the irreducible decomposition

$$
\mathcal{U}\left(\mathfrak{g l}_{n}\right) \cdot \operatorname{det}^{(0)}(X)^{l} \cong \mathcal{S}^{l}\left(\mathbb{C}^{n}\right)^{\otimes n} \cong \bigoplus_{\lambda \vdash l n}\left(\mathcal{M}_{n}^{\lambda}\right)^{\oplus K_{\lambda\left(l^{n}\right)}} .
$$

In general, the module $\mathcal{U}\left(\mathfrak{g l}_{n}\right) \cdot \operatorname{det}^{(\alpha)}(X)^{l}$ is a submodule of $\mathcal{U}\left(\mathfrak{g l}_{n}\right) \cdot \operatorname{det}^{(0)}(X)^{l}$ because $\operatorname{det}^{(\alpha)}(X)^{l} \in \bigoplus_{M \in \mathbb{M}_{n, l}} \mathbb{C} \cdot X^{M}=\mathcal{U}\left(\mathfrak{g l}_{n}\right) \cdot \operatorname{det}^{(0)}(X)^{l}$. Therefore we have

\section{Theorem 3.1.}

$$
\mathcal{U}\left(\mathfrak{g l}_{n}\right) \cdot \operatorname{det}^{(\alpha)}(X)^{l} \cong \bigoplus_{\lambda \vdash l n}\left(\mathcal{M}_{n}^{\lambda}\right)^{\oplus m_{n, l}^{\lambda}(\alpha)},
$$

where $m_{n, l}^{\lambda}(\alpha)$ is a non-negative integer no greater than $K_{\lambda\left(l^{n}\right)}$, with $m_{n, l}^{\lambda}(0)=$ $K_{\lambda\left(l^{n}\right)}$.

In order to obtain further properties of the multiplicities $m_{n, l}^{\lambda}(\alpha)$, we construct a $\mathcal{U}\left(\mathfrak{g l}_{n}\right)$-intertwiner from $\mathcal{S}^{l}\left(\mathbb{C}^{n}\right)^{\otimes n}$ to $\mathcal{U}\left(\mathfrak{g l}_{n}\right) \cdot \operatorname{det}^{(\alpha)}(X)^{l}$ explicitly for each $\alpha$.

For a sequence $\left(k_{1}, \ldots, k_{n}\right) \in[n]^{\times n}$, define

$$
D^{(\alpha)}\left(k_{1}, \ldots, k_{n}\right)=\operatorname{det}^{(\alpha)}\left(\begin{array}{cccc}
x_{k_{1} 1} & x_{k_{1} 2} & \ldots & x_{k_{1} n} \\
x_{k_{2} 1} & x_{k_{2} 2} & \ldots & x_{k_{2} n} \\
\vdots & \vdots & \ddots & \vdots \\
x_{k_{n} 1} & x_{k_{n} 2} & \ldots & x_{k_{n} n}
\end{array}\right) .
$$

For a matrix $N \in \mathbb{M}_{n, 1}$, there exists some $\left(k_{1}, \ldots, k_{n}\right) \in[n]^{\times n}$ such that $N=$ $\left(\delta_{i, k_{j}}\right)_{1 \leq i, j \leq n}$. Then we let

$$
D^{(\alpha)}(N)=D^{(\alpha)}\left(k_{1}, \ldots, k_{n}\right) .
$$

Let $M=\left(m_{i j}\right)_{1 \leq i, j \leq n} \in \mathbb{M}_{n, l}$. A sequence $\left(M_{1}, \ldots, M_{l}\right) \in\left(\mathbb{M}_{n, 1}\right)^{\times l}$ is called a partition of $M$ and denoted by $\left(M_{1}, \ldots, M_{l}\right) \Vdash M$ if $M_{1}+\cdots+M_{l}=M$. We also put $M !=\prod_{i, j=1}^{n} m_{i j} !$. For instance, $\left(l I_{n}\right) !=l !^{n}$. Now we define the element $D^{(\alpha)}(M) \in \mathcal{A}\left(\mathrm{Mat}_{n}\right)$ by

$$
D^{(\alpha)}(M)=\frac{M !}{\left(l I_{n}\right) !} \sum_{\left(M_{1}, \ldots, M_{l}\right) \Vdash M} D^{(\alpha)}\left(M_{1}\right) D^{(\alpha)}\left(M_{2}\right) \cdots D^{(\alpha)}\left(M_{l}\right),
$$

where the sum runs over all partitions of $M$. It is clear that $D^{(\alpha)}\left(l I_{n}\right)=\operatorname{det}^{(\alpha)}(X)^{l}$. 


\section{Example 3.2.}

$$
\begin{aligned}
D^{(\alpha)}\left(\begin{array}{ll}
2 & 1 \\
0 & 1
\end{array}\right) & =D^{(\alpha)}\left(\begin{array}{ll}
1 & 1 \\
0 & 0
\end{array}\right) D^{(\alpha)}\left(\begin{array}{ll}
1 & 0 \\
0 & 1
\end{array}\right)=D^{(\alpha)}(1,1) D^{(\alpha)}(1,2) \\
D^{(\alpha)}\left(\begin{array}{ll}
2 & 1 \\
1 & 2
\end{array}\right)= & \frac{(2 !)^{2}}{(3 !)^{2}}\left\{6 D^{(\alpha)}\left(\begin{array}{ll}
1 & 1 \\
0 & 0
\end{array}\right) D^{(\alpha)}\left(\begin{array}{ll}
1 & 0 \\
0 & 1
\end{array}\right) D^{(\alpha)}\left(\begin{array}{ll}
0 & 0 \\
1 & 1
\end{array}\right)\right. \\
& \left.+3 D^{(\alpha)}\left(\begin{array}{ll}
1 & 0 \\
0 & 1
\end{array}\right)^{2} D^{(\alpha)}\left(\begin{array}{ll}
0 & 1 \\
1 & 0
\end{array}\right)\right\} \\
= & \frac{1}{3}\left(2 D^{(\alpha)}(1,1) D^{(\alpha)}(1,2) D^{(\alpha)}(2,2)+D^{(\alpha)}(1,2)^{2} D^{(\alpha)}(2,1)\right) .
\end{aligned}
$$

Take $M=\left(m_{i j}\right)_{1 \leq i, j \leq n} \in \mathbb{M}_{n, l}$ and suppose that $m_{q k}>0$. Then $M+$ $R_{k}^{p q} \in \mathbb{M}_{n, l}$. Let $\left(M_{1}, \ldots, M_{l}\right) \Vdash M$ and $\left(M_{1}^{\prime}, \ldots, M_{l}^{\prime}\right) \Vdash M+R_{k}^{p q}$. We write $\left(M_{1}, \ldots, M_{l}\right) \stackrel{p, q ; k}{\longrightarrow}\left(M_{1}^{\prime}, \ldots, M_{l}^{\prime}\right)$ if there exists some $j$ such that $M_{i}^{\prime}=M_{i}+\delta_{i j} R_{k}^{p q}$. We notice that

$$
\left|\left\{\left(M_{1}^{\prime}, \ldots, M_{l}^{\prime}\right) \Vdash M+R_{k}^{p q} \mid\left(M_{1}, \ldots, M_{l}\right) \stackrel{p, q ; k}{\longrightarrow}\left(M_{1}^{\prime}, \ldots, M_{l}^{\prime}\right)\right\}\right|=m_{q k}
$$

because $M_{j}+R_{k}^{p q} \in \mathbb{M}_{n, 1}$ if and only if $\left(M_{j}\right)_{q k}=1$ so that the number of such choices of $j$ is just $m_{q k}=\sum_{j=1}^{l}\left(M_{j}\right)_{q k}$. We also notice that

$$
\left(M_{1}, \ldots, M_{l}\right) \stackrel{p, q ; k}{\longrightarrow}\left(M_{1}^{\prime}, \ldots, M_{l}^{\prime}\right) \Longleftrightarrow\left(M_{1}^{\prime}, \ldots, M_{l}^{\prime}\right) \stackrel{q, p ; k}{\longrightarrow}\left(M_{1}, \ldots, M_{l}\right) .
$$

The following fact is crucial.

Proposition 3.3. For any $p, q \in[n]$ and $M \in \mathbb{M}_{n, l}$, we have

$$
E_{p q} \cdot D^{(\alpha)}(M)=\sum_{k=1}^{n} m_{q k} D^{(\alpha)}\left(M+R_{k}^{p q}\right) .
$$

\section{Example 3.4.}

$$
\begin{aligned}
& E_{11} \cdot D^{(\alpha)}\left(\begin{array}{ll}
2 & 1 \\
1 & 2
\end{array}\right)=3 D^{(\alpha)}\left(\begin{array}{ll}
2 & 1 \\
1 & 2
\end{array}\right), \\
& E_{12} \cdot D^{(\alpha)}\left(\begin{array}{ll}
2 & 1 \\
1 & 2
\end{array}\right)=D^{(\alpha)}\left(\begin{array}{ll}
3 & 1 \\
0 & 2
\end{array}\right)+2 D^{(\alpha)}\left(\begin{array}{ll}
2 & 2 \\
1 & 1
\end{array}\right), \\
& E_{21} \cdot D^{(\alpha)}\left(\begin{array}{ll}
2 & 1 \\
1 & 2
\end{array}\right)=2 D^{(\alpha)}\left(\begin{array}{ll}
1 & 1 \\
2 & 2
\end{array}\right)+D^{(\alpha)}\left(\begin{array}{ll}
2 & 0 \\
1 & 3
\end{array}\right), \\
& E_{22} \cdot D^{(\alpha)}\left(\begin{array}{ll}
2 & 1 \\
1 & 2
\end{array}\right)=3 D^{(\alpha)}\left(\begin{array}{ll}
2 & 1 \\
1 & 2
\end{array}\right) .
\end{aligned}
$$


Proof of Proposition 3.3. First we notice that we can verify the case where $l=1$ easily (see Lemma 2.1 in [12]). By using this result, for any $M \in \mathbb{M}_{n, l}$ we have

$$
\begin{aligned}
& E_{p q} \cdot D^{(\alpha)}(M) \\
& =\frac{M !}{\left(l I_{n}\right) !} \sum_{\left(M_{1}, \ldots, M_{l}\right) \Vdash M} \sum_{j=1}^{l} D^{(\alpha)}\left(M_{1}\right) \cdots\left(E_{p q} \cdot D^{(\alpha)}\left(M_{j}\right)\right) \cdots D^{(\alpha)}\left(M_{l}\right) \\
& =\frac{M !}{\left(l I_{n}\right) !} \sum_{k=1}^{n} \sum_{\left(M_{1}, \ldots, M_{l}\right) \Vdash M} \sum_{j=1}^{l}\left(M_{j}\right)_{q k} D^{(\alpha)}\left(M_{1}\right) \cdots D^{(\alpha)}\left(M_{j}+R_{p q}^{k}\right) \cdots D^{(\alpha)}\left(M_{l}\right) \\
& =\frac{M !}{\left(l I_{n}\right) !} \sum_{k=1}^{n} \sum_{\left(M_{1}, \ldots, M_{l}\right) \Vdash M} \sum_{\left(M_{1}^{\prime}, \ldots, M_{l}^{\prime}\right) \Vdash M+R_{k}^{p q}} D^{(\alpha)}\left(M_{1}^{\prime}\right) \cdots D^{(\alpha)}\left(M_{l}^{\prime}\right) \\
& \left(M_{1}, \ldots, M_{l}\right) \stackrel{p, q ; k}{\longrightarrow}\left(M_{1}^{\prime}, \ldots, M_{l}^{\prime}\right) \\
& =\frac{M !}{\left(l I_{n}\right) !} \sum_{k=1}^{n} \sum_{\left(M_{1}^{\prime}, \ldots, M_{l}^{\prime}\right) \Vdash M+R_{k}^{p q}} \sum_{\substack{\left(M_{1}, \ldots, M_{l}\right) \Vdash M \\
\left(M_{1}^{\prime}, \ldots, M_{l}^{\prime}\right) \stackrel{q, p ; k}{\longrightarrow}\left(M_{1}, \ldots, M_{l}\right)}} D^{(\alpha)}\left(M_{1}^{\prime}\right) \ldots D^{(\alpha)}\left(M_{l}^{\prime}\right) .
\end{aligned}
$$

By (3.3), we see that

$$
\begin{aligned}
& \sum_{\substack{\left(M_{1}, \ldots, M_{l}\right) \Vdash M \\
\left(M_{1}^{\prime}, \ldots, M_{l}^{\prime}\right) \stackrel{q, p ; k}{\longrightarrow}\left(M_{1}, \ldots, M_{l}\right)}} 1 \\
= & \left|\left\{\left(M_{1}, \ldots, M_{l}\right) \Vdash\left(M+R_{k}^{p q}\right)+R_{k}^{q p} \mid\left(M_{1}^{\prime}, \ldots, M_{l}^{\prime}\right) \stackrel{q, p ; k}{\longrightarrow}\left(M_{1}, \ldots, M_{l}\right)\right\}\right| \\
= & \left(M+R_{k}^{p q}\right)_{p k}=m_{p k}+1 .
\end{aligned}
$$

Hence it follows that

$$
\begin{aligned}
E_{p q} \cdot D^{(\alpha)}(M) & =\sum_{k=1}^{n}\left(m_{p k}+1\right) \frac{M !}{\left(l I_{n}\right) !} \sum_{\left(M_{1}^{\prime}, \ldots, M_{l}^{\prime}\right) \Vdash M+R_{k}^{p q}} D^{(\alpha)}\left(M_{1}^{\prime}\right) \cdots D^{(\alpha)}\left(M_{l}^{\prime}\right) \\
& =\sum_{k=1}^{n} m_{q k} D^{(\alpha)}\left(M+R_{k}^{p q}\right)
\end{aligned}
$$

since $\left(m_{p k}+1\right) M !=m_{q k}\left(M+R_{k}^{p q}\right) !$ if $m_{q k}>0$. Thus we have proved (3.4).

Now we give an explicit intertwiner from $\mathcal{S}^{l}\left(\mathbb{C}^{n}\right)^{\otimes n}$ to $\mathcal{U}\left(\mathfrak{g l}_{n}\right) \cdot \operatorname{det}^{(\alpha)}(X)^{l}$. The following proposition is a generalization of Lemma 2.3 and Proposition 2.4 in [12] for the case where $l=1$.

Proposition 3.5. We have

$$
\mathcal{U}\left(\mathfrak{g l}_{n}\right) \cdot \operatorname{det}^{(\alpha)}(X)^{l}=\sum_{M \in \mathbb{M}_{n, l}} \mathbb{C} \cdot D^{(\alpha)}(M) .
$$

Furthermore, the linear map $\Phi^{(\alpha)}$ determined by

$$
\Phi^{(\alpha)}\left(\boldsymbol{e}^{M}\right)=D^{(\alpha)}(M) \quad\left(M \in \mathbb{M}_{n, l}\right)
$$

gives a surjective $\mathcal{U}\left(\mathfrak{g l}_{n}\right)$-intertwiner from $\mathcal{S}^{l}\left(\mathbb{C}^{n}\right)^{\otimes n}$ to $\mathcal{U}\left(\mathfrak{g l}_{n}\right) \cdot \operatorname{det}^{(\alpha)}(X)^{l}$. 
Proof. From Proposition 3.3, the space $\sum_{M \in \mathbb{M}_{n, l}} \mathbb{C} \cdot D^{(\alpha)}(M)$ is invariant under the action of $\mathcal{U}\left(\mathfrak{g l}_{n}\right)$. Since $D^{(\alpha)}\left(l I_{n}\right)=\operatorname{det}^{(\alpha)}(X)^{l}$, the space $\mathcal{U}\left(\mathfrak{g l}_{n}\right) \cdot \operatorname{det}^{(\alpha)}(X)^{l}$ is a submodule of $\sum_{M \in \mathbb{M}_{n, l}} \mathbb{C} \cdot D^{(\alpha)}(M)$. Furthermore, by (2.1) and Proposition 3.3. the linear map $\Phi^{(\alpha)}$ determined by

$$
\Phi^{(\alpha)}\left(\boldsymbol{e}^{M}\right)=D^{(\alpha)}(M) \quad\left(M \in \mathbb{M}_{n, l}\right)
$$

gives a surjective $\mathcal{U}\left(\mathfrak{g l}_{n}\right)$-intertwiner from $\mathcal{S}^{l}\left(\mathbb{C}^{n}\right)^{\otimes n}$ to $\sum_{M \in \mathbb{M}_{n, l}} \mathbb{C} \cdot D^{(\alpha)}(M)$. It follows from Lemma 2.2 that

$\sum_{M \in \mathbb{M}_{n, l}} \mathbb{C} \cdot D^{(\alpha)}(M)=\sum_{M \in \mathbb{M}_{n, l}} \mathbb{C} \cdot \Phi^{(\alpha)}\left(e^{M}\right) \subset \mathcal{U}\left(\mathfrak{g l}_{n}\right) \cdot \Phi^{(\alpha)}\left(\boldsymbol{e}^{l I_{n}}\right)=\mathcal{U}\left(\mathfrak{g l}_{n}\right) \cdot \operatorname{det}^{(\alpha)}(X)^{l}$ as desired.

3.2. Transition matrices. We show that the multiplicity $m_{n, l}^{\lambda}(\alpha)$ in Theorem 3.1 is described as a rank of a certain matrix for each highest weight $\lambda$.

The module $\mathcal{S}^{l}\left(\mathbb{C}^{n}\right)^{\otimes n}$ is decomposed in the form

$$
\mathcal{S}^{l}\left(\mathbb{C}^{n}\right)^{\otimes n}=\bigoplus_{\lambda \vdash n l} \bigoplus_{i=1}^{K_{\lambda\left(l^{n}\right)}} \mathcal{U}\left(\mathfrak{g l}_{n}\right) \cdot v_{i}^{\lambda} .
$$

Here $v_{i}^{\lambda}\left(i=1, \ldots, K_{\lambda\left(l^{n}\right)}\right)$ are highest weight vectors corresponding to the weight $\lambda$. Under the isomorphism $\Phi^{(0)}$ and surjective intertwiner $\Phi^{(\alpha)}$, we see that

$$
\begin{aligned}
& \mathcal{U}\left(\mathfrak{g l}_{n}\right) \cdot \operatorname{det}^{(0)}(X)^{l}=\bigoplus_{\lambda \vdash n l} \bigoplus_{i=1}^{K_{\lambda\left(l^{n}\right)}} \mathcal{U}\left(\mathfrak{g l}_{n}\right) \cdot \Phi^{(0)}\left(v_{i}^{\lambda}\right), \\
& \mathcal{U}\left(\mathfrak{g l}_{n}\right) \cdot \operatorname{det}^{(\alpha)}(X)^{l}=\bigoplus_{\lambda \vdash n l} \sum_{i=1}^{K_{\lambda(l n)}} \mathcal{U}\left(\mathfrak{g l}_{n}\right) \cdot \Phi^{(\alpha)}\left(v_{i}^{\lambda}\right) .
\end{aligned}
$$

Since $\Phi^{(\alpha)}\left(v_{i}^{\lambda}\right)$ is the highest weight vector unless it vanishes, there exists a matrix $F_{n, l}^{\lambda}(\alpha)=\left(\left(F_{n, l}^{\lambda}(\alpha)\right)_{i j}\right)$ of size $K_{\lambda\left(l^{n}\right)}$ such that

$$
\Phi^{(\alpha)}\left(v_{j}^{\lambda}\right)=\sum_{i=1}^{K_{\lambda\left(l^{n}\right)}}\left(F_{n, l}^{\lambda}(\alpha)\right)_{i j} \Phi^{(0)}\left(v_{i}^{\lambda}\right)
$$

for each $j$. We call the matrix $F_{n, l}^{\lambda}(\alpha)$ the transition matrix. We notice that the definition of $F_{n, l}^{\lambda}(\alpha)$ is dependent on the choice of vectors $v_{1}^{\lambda}, \ldots, v_{K_{\lambda\left(l^{n}\right)}}^{\lambda}$ but that $F_{n, l}^{\lambda}(\alpha)$ is uniquely determined up to conjugacy. By definition, its entries belong to $\mathbb{Q}[\alpha]$. We now obtain

Theorem 3.6. For each $\alpha \in \mathbb{C}$ and $\lambda \vdash n l$, the multiplicity $m_{n, l}^{\lambda}(\alpha)$ in Theorem 3.1 is equal to the rank of the matrix $F_{n, l}^{\lambda}(\alpha)$ defined via (3.5). Specifically, the irreducible decomposition of the cyclic module $\mathcal{U}\left(\mathfrak{g l}_{n}\right) \cdot \operatorname{det}^{(\alpha)}(X)^{l}$ is given by

$$
\mathcal{U}\left(\mathfrak{g l}_{n}\right) \cdot \operatorname{det}^{(\alpha)}(X)^{l} \cong \bigoplus_{\substack{\lambda \vdash n l \\ \ell(\lambda) \leq n}}\left(\mathcal{M}_{n}^{\lambda}\right)^{\oplus \operatorname{rk} F_{n, l}^{\lambda}(\alpha)} .
$$

We need to obtain an explicit expression for the matrix $F_{n, l}^{\lambda}(\alpha)$ to evaluate the multiplicity $m_{n, l}^{\lambda}(\alpha)$. When $n=2$, we show that the matrix $F_{2, l}^{\lambda}(\alpha)$ is of size 1 and given explicitly by a hypergeometric polynomial. See the next section for a detailed 
discussion of this case. In general, it is not easy to calculate the matrix $F_{n, l}^{\lambda}(\alpha)$, and we have no effective method to evaluate the multiplicity $m_{n, l}^{\lambda}(\alpha)$.

We give several examples of explicit calculation of transition matrices for the highest weights with special types.

Example 3.7. If $l=1$, then we have $F_{n, 1}^{\lambda}(\alpha)=f_{\lambda}(\alpha) I$ for any partition $\lambda$ of $n$, where $f_{\lambda}(\alpha)$ is defined as in (1.3). See Corollary 3.4 in [12].

Example 3.8. For $\lambda=(n l)$, the Kostka number $K_{\lambda\left(l^{n}\right)}$ is equal to 1. The vector $v^{(n l)}=\boldsymbol{e}_{1}^{l} \otimes \boldsymbol{e}_{1}^{l} \otimes \cdots \otimes \boldsymbol{e}_{1}^{l}$ is the highest weight vector with the highest weight $(n l)$. By Proposition 3.5 we have

$$
\begin{aligned}
\Phi^{(\alpha)}\left(v^{(n l)}\right) & =D^{(\alpha)}(1,1, \ldots, 1)^{l} \\
& =\left\{\prod_{j=1}^{n-1}(1+j \alpha) x_{11} x_{22} \cdots x_{n n}\right\}^{l}=\prod_{j=1}^{n-1}(1+j \alpha)^{l} \cdot \Phi^{(0)}\left(v^{(n l)}\right)
\end{aligned}
$$

and hence

$$
F_{n, l}^{(n l)}(\alpha)=\prod_{j=1}^{n-1}(1+j \alpha)^{l}
$$

Example 3.9. For $\lambda=(n l-1,1)$, the Kostka number $K_{\lambda\left(l^{n}\right)}$ is equal to $n-1$. Put

$$
w_{i}=\boldsymbol{e}_{1}^{l} \otimes \boldsymbol{e}_{1}^{l} \otimes \cdots \otimes \boldsymbol{e}_{1}^{l-1} \boldsymbol{e}_{2} \otimes \cdots \otimes \boldsymbol{e}_{1}^{l}
$$

for each $1 \leq i \leq n$. Then $v_{i}^{(n l-1,1)}=w_{i}-w_{i+1}(1 \leq i \leq n-1)$ are linearly independent highest weight vectors corresponding to the weight $(n l-1,1)$. It is easy to see that

$$
\Phi^{(\alpha)}\left(v_{i}^{(n l-1,1)}\right)=(1-\alpha)(1+(n-1) \alpha)^{l-1} \prod_{j=1}^{n-2}(1+j \alpha)^{l} \cdot \Phi^{(0)}\left(v_{i}^{(n l-1,1)}\right),
$$

which readily implies that

$$
F_{n, l}^{(n l-1,1)}(\alpha)=(1-\alpha)(1+(n-1) \alpha)^{l-1} \prod_{j=1}^{n-2}(1+j \alpha)^{l} \cdot I_{n-1} .
$$

\section{4. $\mathcal{U}\left(\mathfrak{g l}_{2}\right)$-CYCLIC MODULES AND JACOBI POLYNOMIALS}

In this section, we study the case where $n=2$. The transition matrix $F_{2, l}^{\lambda}(\alpha)$ is of size 1 and explicitly given by a hypergeometric polynomial in $\alpha$ which is in fact the Jacobi polynomial. Moreover, we see that these Jacobi polynomials are unitary.

4.1. Explicit irreducible decomposition of $\mathcal{U}\left(\mathfrak{g l}_{n}\right) \cdot \operatorname{det}^{(\alpha)}(X)^{l}$. For a nonnegative integer $n$ and complex numbers $b$ and $c$ such that $c \neq-1,-2, \ldots,-n+1$, let $F(-n, b, c ; x)$ be the Gaussian hypergeometric polynomial

$$
F(-n, b, c ; x)=1+\sum_{k=1}^{n} \frac{(-n)_{k}(b)_{k}}{(c)_{k}} \frac{x^{k}}{k !} .
$$

Here $(a)_{k}$ stands for the Pochhammer symbol $(a)_{k}=a(a+1) \cdots(a+k-1)$. For any partition $\lambda$ of $2 l$ with length $\leq 2$, we have $K_{\lambda\left(l^{2}\right)}=1$, whence $F_{2, l}^{\lambda}(\alpha)$ is a scalar. 
Theorem 4.1. For non-negative integers $l$ and $s$ such that $0 \leq s \leq l$, we have

$$
F_{2, l}^{(2 l-s, s)}(\alpha)=(1+\alpha)^{l-s} G_{s}^{l}(\alpha),
$$

where $G_{n}^{\gamma}(x)$ is the polynomial given by

$$
G_{n}^{\gamma}(x)=F(-n, \gamma-n+1,-\gamma ;-x) .
$$

By the hypergeometric differential equation satisfied by $G_{n}^{\gamma}(x)$, the explicit form of $F_{2, l}^{(2 l-s, s)}(\alpha)$ given in Theorem 4.1 shows that $F_{2, l}^{(2 l-s, s)}(\alpha)$ satisfies the following singly confluent Heun differential equation (see [14]).

Corollary 4.2. The polynomial $f(x)=F_{2, l}^{(2 l-s, s)}(-x)$ satisfies the differential equation

$$
\left\{\frac{d^{2}}{d x^{2}}+\left(\frac{2}{x-1}-\frac{l}{x}\right) \frac{d}{d x}+\frac{s-(l-s)^{2}-x}{x(x-1)^{2}}\right\} f(x)=0 .
$$

Remark 4.3. Since it seems difficult at present to obtain the transition matrices explicitly in general, we are naturally led to the following questions: Can one obtain equation (4.2) directly by investigating (a certain structure of) the cyclic module $\mathcal{U}\left(\mathfrak{g l}_{2}\right) \cdot \operatorname{det}^{(\alpha)}(X)^{l}$ itself? If this is possible, can the derivation of the differential equation then be generalized to the cases where $n \geq 3$ ?

The roots of the polynomial $G_{n}^{\gamma}(x)$ satisfy the following property.

Proposition 4.4. For a real number $\gamma$ such that $\gamma \geq n$, the polynomial $G_{n}^{\gamma}(x)$ is unitary, i.e., every root of $G_{n}^{\gamma}(x)$ is on the unit circle $\mathbb{T}=\{z \in \mathbb{C}|| z \mid=1\}$. Furthermore, $G_{2 n}^{\gamma}(1) \neq 0$ and $G_{2 n+1}^{\gamma}(1)=0$ for any non-negative integer $n$.

Therefore we obtain the following irreducible decomposition from Theorem 3.1 and Proposition 3.6 .

Corollary 4.5. For any $\alpha \in \mathbb{C} \backslash \mathbb{T}$, we have

$$
\mathcal{U}\left(\mathfrak{g l}_{2}\right) \cdot \operatorname{det}^{(\alpha)}(X)^{l} \cong \mathcal{S}^{l}\left(\mathbb{C}^{2}\right)^{\otimes 2} \cong \bigoplus_{s=0}^{l} \mathcal{M}_{2}^{(2 l-s, s)} .
$$

For $\alpha= \pm 1$, we have

$$
\begin{aligned}
& \mathcal{U}\left(\mathfrak{g l}_{2}\right) \cdot \operatorname{per}(X)^{l} \cong \bigoplus_{j=0}^{\lfloor l / 2\rfloor} \mathcal{M}_{2}^{(2 l-2 j, 2 j)} \cong \mathcal{S}^{l}\left(\mathcal{S}^{2}\left(\mathbb{C}^{2}\right)\right), \\
& \mathcal{U}\left(\mathfrak{g l}_{2}\right) \cdot \operatorname{det}(X)^{l}=\mathbb{C} \cdot \operatorname{det}(X)^{l} \cong \mathcal{M}_{2}^{(l, l)}
\end{aligned}
$$

4.2. Proof of Theorem 4.1, The highest weight vector associated with the highest weight $(2 l-s, s)$ in the module $\mathcal{S}^{l}\left(\mathbb{C}^{2}\right)^{\otimes 2}\left(\cong \bigoplus_{s=0}^{l} \mathcal{M}_{2}^{(2 l-s, s)}\right)$ is given by

$$
v^{(2 l-s, s)}=\sum_{j=0}^{s}(-1)^{j}\left(\begin{array}{l}
s \\
j
\end{array}\right) \boldsymbol{e}_{1}^{l-j} \boldsymbol{e}_{2}^{j} \otimes \boldsymbol{e}_{1}^{l-s+j} \boldsymbol{e}_{2}^{s-j} .
$$

The image of this under $\Phi^{(\alpha)}$ is

$$
\Phi^{(\alpha)}\left(v^{(2 l-s, s)}\right)=\sum_{j=0}^{s}(-1)^{j}\left(\begin{array}{l}
s \\
j
\end{array}\right) D^{(\alpha)}\left(\begin{array}{cc}
l-j & l-s+j \\
j & s-j
\end{array}\right) .
$$


Lemma 4.6. For $0 \leq p \leq q \leq l$, we have

$$
\begin{aligned}
D^{(\alpha)}\left(\begin{array}{cc}
l-p & l-q \\
p & q
\end{array}\right) & =\left(\begin{array}{l}
l \\
q
\end{array}\right)^{-1} \sum_{r=0}^{\min \{p, l-q\}}\left(\begin{array}{c}
l-p \\
q-p+r
\end{array}\right)\left(\begin{array}{l}
p \\
r
\end{array}\right) \\
& \times D^{(\alpha)}(1,1)^{l-q-r} D^{(\alpha)}(1,2)^{q-p+r} D^{(\alpha)}(2,1)^{r} D^{(\alpha)}(2,2)^{p-r} .
\end{aligned}
$$

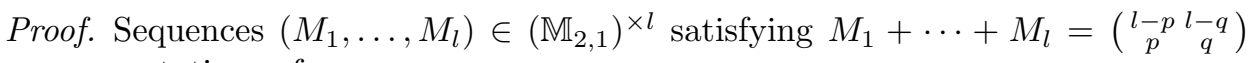
are permutations of

$$
\begin{aligned}
& \overbrace{\left(\begin{array}{ll}
1 & 1 \\
0 & 0
\end{array}\right), \ldots,\left(\begin{array}{ll}
1 & 1 \\
0 & 0
\end{array}\right)}^{l-q-r}, \overbrace{\left(\begin{array}{ll}
1 & 0 \\
0 & 1
\end{array}\right), \ldots,\left(\begin{array}{ll}
1 & 0 \\
0 & 1
\end{array}\right)}^{q-p+r}, \\
& \overbrace{\left(\begin{array}{ll}
0 & 1 \\
1 & 0
\end{array}\right), \ldots,\left(\begin{array}{ll}
0 & 1 \\
1 & 0
\end{array}\right)}^{r}, \overbrace{\left(\begin{array}{ll}
0 & 0 \\
1 & 1
\end{array}\right), \ldots,\left(\begin{array}{ll}
0 & 0 \\
1 & 1
\end{array}\right)}^{p-r},
\end{aligned}
$$

where $r$ runs over $0,1, \ldots, \min \{p, l-q\}$. Since the number of such sequences is $(l !) /\{(l-q-r) !(q-p+r) !(p-r) !\}$, we have

$$
\begin{aligned}
& D^{(\alpha)}\left(\begin{array}{cc}
l-p & l-q \\
p & q
\end{array}\right) \\
& \quad=\frac{(l-p) !(l-q) ! p ! q !}{(l !)^{2}} \sum_{r=0}^{\min \{p, l-q\}} \frac{l !}{(l-q-r) !(q-p+r) ! r !(p-r) !} \\
& \quad \times D^{(\alpha)}(1,1)^{l-q-r} D^{(\alpha)}(1,2)^{q-p+r} D^{(\alpha)}(2,1)^{r} D^{(\alpha)}(2,2)^{p-r} .
\end{aligned}
$$

This completes the proof.

The polynomial $F_{2, l}^{(2 l-s, s)}(\alpha)$ is determined by the identity $\Phi^{(\alpha)}\left(v^{(2 l-s, s)}\right)=$ $F_{2, l}^{(2 l-s, s)}(\alpha) \Phi^{(0)}\left(v^{(2 l-s, s)}\right)$. By comparing the coefficients of $x_{11}^{l} x_{12}^{l-s} x_{22}^{s}$ in the two sides, we see that

$$
F_{2, l}^{(2 l-s, s)}(\alpha)=\left[x_{11}^{l} x_{12}^{l-s} x_{22}^{s}\right] \Phi^{(\alpha)}\left(v^{(2 l-s, s)}\right) .
$$

Here $\left[x_{11}^{a} x_{12}^{b} x_{21}^{c} x_{22}^{d}\right] f\left(x_{11}, x_{12}, x_{21}, x_{22}\right)$ stands for the coefficient of $x_{11}^{a} x_{12}^{b} x_{21}^{c} x_{22}^{d}$ in $f\left(x_{11}, x_{12}, x_{21}, x_{22}\right)$. By using Lemma 4.6 together with

$$
\begin{array}{ll}
D^{(\alpha)}(1,1)=(1+\alpha) x_{11} x_{12}, & D^{(\alpha)}(1,2)=x_{11} x_{22}+\alpha x_{21} x_{12}, \\
D^{(\alpha)}(2,1)=\alpha x_{11} x_{22}+x_{21} x_{12}, & D^{(\alpha)}(2,2)=(1+\alpha) x_{21} x_{22},
\end{array}
$$

we have

$$
\begin{aligned}
& {\left[x_{11}^{l} x_{12}^{l-s} x_{22}^{s}\right] D^{(\alpha)}\left(\begin{array}{cc}
l-j & l-s+j \\
j & s-j
\end{array}\right)} \\
& =\left(\begin{array}{c}
l \\
s-j
\end{array}\right)^{-1}\left(\begin{array}{l}
l-j \\
s-j
\end{array}\right) \\
& \times\left(\left[\left(x_{11} x_{12}\right)^{l-s}\right] D^{(\alpha)}(1,1)^{l-s}\right) \cdot\left(\left[\left(x_{11} x_{22}\right)^{s}\right] D^{(\alpha)}(1,2)^{s-j} D^{(\alpha)}(2,1)^{j}\right) \\
& =\frac{(l-j) !(l-s+j) !}{l !(l-s) !}(1+\alpha)^{l-s} \alpha^{j}
\end{aligned}
$$


for $0 \leq j<s / 2$. We can check that this identity holds for any $0 \leq j \leq s$ in a similar way. Hence it follows from (4.3) that

$$
\begin{aligned}
F_{2, l}^{(2 l-s, s)}(\alpha) & =\frac{s !}{l !(l-s) !}(1+\alpha)^{l-s} \sum_{j=0}^{s} \frac{(l-j) !(l-s+j) !}{(s-j) !} \cdot \frac{(-\alpha)^{j}}{j !} \\
& =(1+\alpha)^{l-s} \sum_{j=0}^{s} \frac{s !(l-j) !(l-s+j) !}{l !(l-s) !(s-j) !} \cdot \frac{(-\alpha)^{j}}{j !} \\
& =(1+\alpha)^{l-s} F(-s, l-s+1,-l ;-\alpha) .
\end{aligned}
$$

Thus we have proved Theorem 4.1 .

4.3. Proof of Proposition 4.4. Let $\gamma$ be a positive real number and $n$ a nonnegative integer such that $\gamma \geq n$. We prove the unitarity of the polynomial $G_{n}^{\gamma}(x)$ by using properties of the Jacobi polynomial (see e.g. [15])

$$
P_{n}^{(\alpha, \beta)}(x)=\left(\begin{array}{c}
n+\alpha \\
n
\end{array}\right) F\left(-n, n+\alpha+\beta+1, \alpha+1 ; \frac{1-x}{2}\right) .
$$

We see by definition that

$$
G_{n}^{\gamma}(x)=\left(\begin{array}{c}
n-\gamma-1 \\
n
\end{array}\right)^{-1} P_{n}^{(-\gamma-1,2 \gamma-2 n+1)}(1+2 x) .
$$

We recall the following formulas ((4.1.3), (4.22.1), and (4.1.5) in [15]).

Lemma 4.7. For any $\alpha, \beta \in \mathbb{C}$ and non-negative integer $n$, the following formulas hold.

$$
\begin{aligned}
& P_{n}^{(\alpha, \beta)}(x)=(-1)^{n} P_{n}^{(\beta, \alpha)}(-x), \\
& P_{n}^{(\alpha, \beta)}(x)=\left(\frac{1-x}{2}\right)^{n} P_{n}^{(-2 n-\alpha-\beta-1, \beta)}\left(\frac{x+3}{x-1}\right), \\
& P_{2 n}^{(\alpha, \beta)}(x)=\frac{(-1)^{n} n !}{(2 n) !}(\alpha+n+1)_{n} P_{n}^{\left(-\frac{1}{2}, \alpha\right)}\left(1-2 x^{2}\right), \\
& P_{2 n+1}^{(\alpha, \beta)}(x)=\frac{(-1)^{n} n !}{(2 n+1) !}(\alpha+n+1)_{n+1} x P_{n}^{\left(\frac{1}{2}, \alpha\right)}\left(1-2 x^{2}\right) .
\end{aligned}
$$

From (4.4) and (4.5), we have

$$
G_{n}^{\gamma}(x)=\left(\begin{array}{c}
n-\gamma-1 \\
n
\end{array}\right)^{-1}(-1)^{n} P_{n}^{(2 \gamma-2 n+1,-\gamma-1)}(-1-2 x) .
$$

From (4.6), it follows that

$$
G_{n}^{\gamma}(x)=\left(\begin{array}{c}
n-\gamma-1 \\
n
\end{array}\right)^{-1}(-1)^{n}(1+x)^{n} P_{n}^{(-\gamma-1,-\gamma-1)}\left(\frac{x-1}{x+1}\right) .
$$

Applying (4.7) and (4.8) to this expression, we obtain the following lemma. 


\section{Lemma 4.8.}

$$
\begin{aligned}
G_{2 m}^{\gamma}(x) & =\frac{(-1)^{m} m !}{(-\gamma)_{m}}(x+1)^{2 m} P_{m}^{(-1 / 2,-\gamma-1)}\left(1-2\left(\frac{x-1}{x+1}\right)^{2}\right), \\
G_{2 m+1}^{\gamma}(x) & =\frac{(-1)^{m+1} m !}{(-\gamma)_{m}}(x-1)(x+1)^{2 m} P_{m}^{(1 / 2,-\gamma-1)}\left(1-2\left(\frac{x-1}{x+1}\right)^{2}\right) .
\end{aligned}
$$

In particular, $G_{2 m}^{\gamma}(1) \neq 0$ and $G_{2 m+1}^{\gamma}(1)=0$.

In general, the distribution of the roots of Jacobi polynomials are described as follows.

Lemma 4.9 (Theorem 6.72 in [15]). For any real number $u$, let $E(u)$ be the Klein symbol, i.e.,

$$
E(u)= \begin{cases}u-1 & \text { if } u \text { is an integer and } u \geq 0, \\ \lfloor u\rfloor & \text { if } u \text { is not an integer and } u \geq 0, \\ 0 & \text { if } u<0 .\end{cases}
$$

Let $\alpha$ and $\beta$ be complex numbers and $n$ a non-negative integer. Assume $\prod_{k=1}^{n}(\alpha+$ $k)(\beta+k)(n+\alpha+\beta+k) \neq 0$. Define three numbers $X, Y$, and $Z$ by

$$
\begin{aligned}
& X=E\left(\frac{1}{2}(|2 n+\alpha+\beta+1|-|\alpha|-|\beta|+1)\right), \\
& Y=E\left(\frac{1}{2}(-|2 n+\alpha+\beta+1|+|\alpha|-|\beta|+1)\right), \\
& Z=E\left(\frac{1}{2}(-|2 n+\alpha+\beta+1|-|\alpha|+|\beta|+1)\right) .
\end{aligned}
$$

Then, if we denote by $N(I)$ the number of roots of $P_{n}^{(\alpha, \beta)}(x)$ in an interval $I \subset \mathbb{R}$, we have

$$
\begin{gathered}
N((-1,1))= \begin{cases}2\lfloor(X+1) / 2\rfloor, & \text { if }(-1)^{n}\left(\begin{array}{c}
n+\alpha \\
n
\end{array}\right)\left(\begin{array}{c}
n+\beta \\
n
\end{array}\right)>0, \\
2\lfloor X / 2\rfloor+1, & \text { if }(-1)^{n}\left(\begin{array}{c}
n+\alpha \\
n
\end{array}\right)\left(\begin{array}{c}
n+\beta \\
n
\end{array}\right)<0,\end{cases} \\
N((-\infty,-1))= \begin{cases}2\lfloor(Y+1) / 2\rfloor, & \text { if }\left(\begin{array}{c}
2 n+\alpha+\beta \\
n
\end{array}\right)\left(\begin{array}{c}
n+\beta \\
n
\end{array}\right)>0, \\
2\lfloor Y / 2\rfloor+1, & \text { if }\left(\begin{array}{c}
2 n+\alpha+\beta \\
n
\end{array}\right)\left(\begin{array}{c}
n+\beta \\
n
\end{array}\right)<0,\end{cases} \\
N((1, \infty))= \begin{cases}2\lfloor(Z+1) / 2\rfloor, & \text { if }\left(\begin{array}{c}
2 n+\alpha+\beta \\
n
\end{array}\right)\left(\begin{array}{c}
n+\alpha \\
n
\end{array}\right)>0, \\
2\lfloor Z / 2\rfloor+1, & \text { if }\left(\begin{array}{c}
2 n+\alpha+\beta \\
n
\end{array}\right)\left(\begin{array}{c}
n+\alpha \\
n
\end{array}\right)<0 .\end{cases}
\end{gathered}
$$

Let us set $\alpha=-1 / 2, \beta=-\gamma-1$ and $n=2 m$ (resp. $\alpha=1 / 2, \beta=-\gamma-1$ and $n=2 m+1)$ in the lemma above and assume that $\gamma \geq n$. It follows that $X=Y=0$ and $Z=m$, from which we have $N((-1,1))=N((-\infty,-1))=0$ and $N((1, \infty))=m$. Since the degree of the polynomial $P_{m}^{(-1 / 2,-\gamma-1)}(x)$ (resp. $\left.P_{m}^{(1 / 2,-\gamma-1)}(x)\right)$ is $m$, all roots of $P_{m}^{(-1 / 2,-\gamma-1)}(x)$ (resp. $P_{m}^{(1 / 2,-\gamma-1)}(x)$ ) belong to the interval $(1, \infty)$. Therefore it follows from Lemma 4.8 that

$$
a \in \mathbb{C}, \quad G_{n}^{\gamma}(a)=0 \quad \Longrightarrow \quad \frac{a-1}{a+1} \in i \mathbb{R} \quad \Longrightarrow \quad|a|=1 .
$$

This completes the proof of Proposition 4.4 


\section{Several Remarks on problems for future Study}

We give here several comments on cases for future study.

5.1. Permanent cases. When $\alpha=-1$, $\operatorname{det}^{(-1)}(X)$ is just the ordinary determinant and we can easily see that the cyclic module $\mathcal{U}\left(\mathfrak{g l}_{n}\right) \cdot \operatorname{det}^{(-1)}(X)^{l}$ is isomorphic to $\mathcal{M}_{n}^{\left(l^{n}\right)}$. However, in the case where $\alpha=1$, we have not obtained the irreducible decomposition of the cyclic module $\mathcal{U}\left(\mathfrak{g l}_{n}\right) \cdot \operatorname{det}^{(1)}(X)^{l}$ generated by the permanent $\operatorname{per}(X)=\operatorname{det}^{(1)}(X)$. Actually, all we can give here is the following conjecture.

Conjecture 5.1. $\mathcal{U}\left(\mathfrak{g l}_{n}\right) \cdot \operatorname{det}^{(1)}(X)^{l} \cong \mathcal{S}^{l}\left(\mathcal{S}^{n}\left(\mathbb{C}^{n}\right)\right)$.

This claim is equivalent to the assertion that the character of $\mathcal{U}\left(\mathfrak{g l}_{n}\right) \cdot \operatorname{det}^{(1)}(X)^{l}$ is given by the plethysm $h_{l} \circ h_{n}$. (For the definition of the plethysm for symmetric functions, see [10, Chapter I-8].) We have already verified this conjecture in the following cases: (i) $l=1$ (see [12]), (ii) $n=1,2$ (see the previous section), (iii) $n=3$ and $l=2$ (see Example 5.2 below).

Example 5.2. Let $n=3$ and $l=2$. If we take suitable highest weight vectors and employ a similar calculation as in the proof of Theorem 4.1, we have

$$
\begin{aligned}
F_{3,2}^{(6)}(\alpha) & =(1+\alpha)^{2}(1+2 \alpha)^{2}, \\
F_{3,2}^{(5,1)}(\alpha) & =(1-\alpha)(1+\alpha)^{2}(1+2 \alpha) I_{2}, \\
F_{3,2}^{(4,2)}(\alpha) & =(1+\alpha)^{2} \cdot \operatorname{diag}\left(2(1-\alpha), 2(1-\alpha), 2-2 \alpha+3 \alpha^{2}\right), \\
F_{3,2}^{(4,1,1)}(\alpha) & =\frac{1}{2}(1-\alpha)(1+\alpha)\left(2-5 \alpha^{2}\right), \\
F_{3,2}^{(3,3)}(\alpha) & =(1-\alpha)^{2}\left(1+\alpha^{2}\right), \\
F_{3,2}^{(3,2,1)}(\alpha) & =\frac{1}{4}(1-\alpha)(1+\alpha)\left(4-6 \alpha+5 \alpha^{2}\right) I_{2}, \\
F_{3,2}^{(2,2,2)}(\alpha) & =\frac{1}{2}(1-\alpha)^{2}\left(2-2 \alpha+5 \alpha^{2}\right) .
\end{aligned}
$$

In particular, when $\alpha=1$ we see that

$$
m_{3,2}^{\lambda}(1)= \begin{cases}1 & \text { if } \lambda=(6),(4,2), \\ 0 & \text { otherwise }\end{cases}
$$

and hence it follows that

$$
\mathcal{U}\left(\mathfrak{g l}_{3}\right) \cdot \operatorname{per}(X)^{2} \cong \mathcal{M}_{3}^{(6)} \oplus \mathcal{M}_{3}^{(4,2)} \cong \mathcal{S}^{2}\left(\mathcal{S}^{3}\left(\mathbb{C}^{3}\right)\right),
$$

which agrees with our conjecture.

5.2. Complex powers of $\alpha$-determinants. An appropriate reformulation of the setting is necessary to study "the cyclic module $\mathcal{U}\left(\mathfrak{g l}_{n}\right) \cdot \operatorname{det}^{(\alpha)}(X)^{s}$ " with a complex number $s$. Here we introduce a suitable space in which we can treat such cyclic modules.

We take a $\mathcal{U}\left(\mathfrak{g l}_{n}\right)$-submodule

$$
\mathrm{ML}_{n}^{\cdot}=\left\{F_{1} \cdots F_{k} \mid k \geq 0, F_{i} \in \bigoplus_{M \in \mathbb{M}_{n, 1}} \mathbb{C} \cdot X^{M}\right\}
$$


of $\mathcal{A}\left(\mathrm{Mat}_{n}\right)$, and consider the tensor product

$$
\mathrm{ML}_{n}^{\bullet} \otimes_{\mathbb{C}}\left(\bigoplus_{k=0}^{\infty} \mathbb{C} \cdot w(\alpha, s-k)\right)
$$

where $\{w(\alpha, s-k)\}_{k \geq 0}$ are formal vectors. We introduce a $\mathcal{U}\left(\mathfrak{g l}_{n}\right)$-module structure on it by defining

$Y \cdot(F \otimes w(\alpha, s-k))=(Y \cdot F) \otimes w(\alpha, s-k)+(s-k) F\left(Y \cdot \operatorname{det}^{(\alpha)}(X)\right) \otimes w(\alpha, s-k-1)$

for $Y \in \mathfrak{g l}_{n}$ and $F \in \mathrm{ML}_{n}^{\bullet}$. Let $\mathcal{M L}_{n}(\alpha, s)$ be the quotient $\mathcal{U}\left(\mathfrak{g l}_{n}\right)$-module of $\mathrm{ML}_{n}^{\bullet} \otimes_{\mathbb{C}}\left(\bigoplus_{k=0}^{\infty} \mathbb{C} \cdot w(\alpha, s-k)\right)$ with respect to the submodule generated by

$$
\left(F \cdot \operatorname{det}^{(\alpha)}(X)\right) \otimes w(\alpha, s-k)-F \otimes w(\alpha, s-k+1) \quad\left(F \in \mathrm{ML}_{n}^{\bullet}, w(\alpha, 0)=1\right) .
$$

For $F(X) \in \mathrm{ML}_{n}^{\bullet}$ and $k \in \mathbb{Z}_{+}$, we denote by $F(X) \operatorname{det}^{(\alpha)}(X)^{s-k}$ the element in $\mathcal{M L}_{n}(\alpha, s)$ represented by $F(X) \otimes w(\alpha, s-k)$. We notice that the relation $\operatorname{det}^{(\alpha)}(X) \operatorname{det}^{(\alpha)}(X)^{s-k}=\operatorname{det}^{(\alpha)}(X)^{s-k+1}$ holds by (5.2).

Let us denote by $\mathcal{V}(\alpha, s)$ the submodule of $\mathcal{M L}_{n}(\alpha, s)$ generated by the vector $\operatorname{det}^{(\alpha)}(X)^{s}(=1 \otimes w(\alpha, s))$. When $s$ is a non-negative integer $l$, we can naturally consider that

$$
\mathcal{M L}_{n}(\alpha, l) \subset \mathrm{ML}_{n}^{\bullet}
$$

because $1 \otimes w(\alpha, l)=\operatorname{det}^{(\alpha)}(X)^{l} \otimes 1$, so that

$$
\mathcal{V}(\alpha, l) \cong \mathcal{U}\left(\mathfrak{g l}_{n}\right) \cdot \operatorname{det}^{(\alpha)}(X)^{l} .
$$

Thus we regard the space $\mathcal{V}(\alpha, s)$ as a suitable formulation of the cyclic module $\mathcal{U}\left(\mathfrak{g l}_{n}\right) \cdot \operatorname{det}^{(\alpha)}(X)^{s}$ for $s \in \mathbb{C}$. We note that $\mathcal{M} \mathcal{L}_{n}(\alpha, l)$ can be realized in the quotient field for the algebra $\mathrm{ML}_{n}^{\bullet}$ when $l$ is a negative integer.

Example 5.3. Let $\alpha=-1$. Then $\mathcal{V}(-1, s)=\mathcal{U}\left(\mathfrak{g l}_{n}\right) \cdot \operatorname{det}(X)^{s}$ is a one-dimensional space and we have $E_{p p} \cdot \operatorname{det}^{(-1)}(X)^{s}=s \operatorname{det}^{(-1)}(X)^{s}$ for any $1 \leq p \leq n$ from (5.1). Thus the module $\mathcal{V}(-1, s)$ is the irreducible module with "highest weight $(s, s, \ldots, s)$ ".

The module $\mathcal{V}(\alpha, s)$ is infinite dimensional in general. For instance, if $\alpha=0$ and $s \in \mathbb{C} \backslash \mathbb{Z}_{+}$, then we see that

$$
E_{12}^{k} \cdot \operatorname{det}^{(0)}(X)^{s}=s(s-1) \cdots(s-k+1)\left(x_{11} x_{12} x_{33} x_{44} \cdots x_{n n}\right)^{k} \operatorname{det}^{(0)}(X)^{s-k}
$$

for each $k \geq 0$ and these vectors are linearly independent; this obviously implies that $\operatorname{dim}_{\mathbb{C}} \mathcal{V}(0, s)=\infty$. In the infinite dimensional cases, the following two problems are fundamental:

(1) Unitarizability of each irreducible subrepresentation appearing in the decomposition of the cyclic module $\mathcal{U}\left(\mathfrak{g l}_{n}\right) \cdot \operatorname{det}^{(\alpha)}(X)^{s}$.

(2) Description of the "content function" for each isotypic component in $\mathcal{U}\left(\mathfrak{g l}_{n}\right)$. $\operatorname{det}^{(\alpha)}(X)^{s}$ as a certain special function such as a solution of some Fuchsian type ordinary differential equation. (See Remark 4.3.)

We will treat these problems in our future studies. 
5.3. Generalized immanants. Let $\varphi$ be a class function on $\mathfrak{S}_{n}$. We define the $\varphi$-immanant by

$$
\operatorname{imm}^{\varphi}(X)=\sum_{\sigma \in \mathfrak{S}_{n}} \varphi(\sigma) x_{1 \sigma(1)} \cdots x_{n \sigma(n)} .
$$

For $l$ class functions $\varphi_{1}, \ldots, \varphi_{l}$, consider the cyclic module

$$
\mathcal{U}\left(\mathfrak{g l}_{n}\right) \cdot \prod_{i=1}^{l} \operatorname{imm}^{\varphi_{i}}(X),
$$

which is a submodule of $\bigoplus_{M \in \mathbb{M}_{n, l}} \mathbb{C} \cdot X^{M}$. In this article we have discussed the special case where $\varphi_{1}(\sigma)=\cdots=\varphi_{l}(\sigma)=\alpha^{\nu(\sigma)}$. The discussion, and hence several propositions, in Section 3 can be extended to this generalized situation because we do not use any special feature of the function $\alpha^{\nu(\sigma)}$. See the Appendix below.

\section{Appendix: TRANSITION MATRICES AND zONAL SPHERICAL FUnCtions By Kazufumi Кiмото}

We investigate the structure of the cyclic module $\boldsymbol{V}_{n, l}(\alpha)=\mathcal{U}\left(\mathfrak{g l}_{n}\right) \cdot \operatorname{det}^{(\alpha)}(X)^{l}$ by embedding it into the tensor product space $\left(\mathbb{C}^{n}\right)^{\otimes n l}$ and utilizing the Schur-Weyl duality. We show that the entries of the transition matrices $F_{n, l}^{\lambda}(\alpha)$ are given by a variation of the spherical Fourier transformation of a certain class function on $\mathfrak{S}_{n l}$ with respect to the subgroup $\mathfrak{S}_{l}^{n}$ (Theorem 6.4). This result also provides another proof of Theorem 3.6. Further, we calculate the polynomial $F_{2, l}^{(2 l-s, s)}(\alpha)$ by using an explicit formula for the values of zonal spherical functions for the Gelfand pair $\left(\mathfrak{S}_{2 l}, \mathfrak{S}_{l} \times \mathfrak{S}_{l}\right)$ due to Bannai and Ito (Theorem 6.11).

6.1. Irreducible decomposition of $\boldsymbol{V}_{n, l}(\alpha)$ and transition matrices. Let us fix $n, l \in \mathbb{N}$. Consider the standard tableau $\mathbb{T}$ with shape $\left(l^{n}\right)$ such that the $(i, j)$ entry of $\mathbb{T}$ is $(i-1) l+j$. For instance, if $n=3$ and $l=2$, then

$$
\mathbb{T}=\begin{array}{|l|l|}
\hline 1 & 2 \\
\hline 3 & 4 \\
\hline 5 & 6 \\
\hline
\end{array}
$$

We denote by $K=R(\mathbb{T})$ and $H=C(\mathbb{T})$ the row group and column group of the standard tableau $\mathbb{T}$ respectively, that is,

$$
\begin{aligned}
& K=\left\{g \in \mathfrak{S}_{n l} \mid\lceil g(x) / l\rceil=\lceil x / l\rceil, x \in[n l]\right\}, \\
& H=\left\{g \in \mathfrak{S}_{n l} \mid g(x) \equiv x \quad(\bmod l), x \in[n l]\right\} .
\end{aligned}
$$

We put

$$
e=\frac{1}{|K|} \sum_{k \in K} k \in \mathbb{C}\left[\mathfrak{S}_{n l}\right]
$$

This is clearly an idempotent element in $\mathbb{C}\left[\mathfrak{S}_{n l}\right]$. Let $\varphi$ be a class function on $H$. We put

$$
\Phi=\sum_{h \in H} \varphi(h) h \in \mathbb{C}\left[\mathfrak{S}_{n l}\right] .
$$


Consider the tensor product space $V=\left(\mathbb{C}^{n}\right)^{\otimes n l}$. We introduce a $\left(\mathcal{U}\left(\mathfrak{g l}_{n}\right), \mathbb{C}\left[\mathfrak{S}_{n l}\right]\right)$ module structure of $V$ by writing

$$
\begin{aligned}
E_{i j} \cdot \boldsymbol{e}_{i_{1}} \otimes \cdots \otimes \boldsymbol{e}_{i_{n l}} & =\sum_{s=1}^{n l} \delta_{i_{s}, j} \boldsymbol{e}_{i_{1}} \otimes \cdots \otimes \boldsymbol{e}_{i} \otimes \cdots \otimes \boldsymbol{e}_{i_{n l}}, t h \\
\boldsymbol{e}_{i_{1}} \otimes \cdots \otimes \boldsymbol{e}_{i_{n l}} \cdot \sigma & =\boldsymbol{e}_{i_{\sigma(1)}} \otimes \cdots \otimes \boldsymbol{e}_{i_{\sigma(n l)}} \quad\left(\sigma \in \mathfrak{S}_{n l}\right),
\end{aligned}
$$

where $\left\{\boldsymbol{e}_{i}\right\}_{i=1}^{n}$ denotes the standard basis of $\mathbb{C}^{n}$. The main concern of this subsection is to describe the irreducible decomposition of the left $\mathcal{U}\left(\mathfrak{g l}_{n}\right)$-module $V \cdot e \Phi e$.

We first show that $\boldsymbol{V}_{n, l}(\alpha)$ is isomorphic to $V \cdot e \Phi e$ for a special choice of $\varphi$. Consider the group isomorphism $\theta: H \rightarrow \mathfrak{S}_{n}^{l}$ defined by

$$
\theta(h)=\left(\theta(h)_{1}, \ldots, \theta(h)_{l}\right) ; \quad \theta(h)_{i}(x)=y \Longleftrightarrow h((x-1) l+i)=(y-1) l+i .
$$

We also define an element $D(X ; \varphi) \in \mathcal{A}\left(\mathrm{Mat}_{n}\right)$ by

$$
\begin{aligned}
D(X ; \varphi) & =\sum_{h \in H} \varphi(h) \prod_{q=1}^{n} \prod_{p=1}^{l} x_{\theta(h)_{p}(q), q}=\sum_{h \in H} \varphi(h) \prod_{q=1}^{n} \prod_{p=1}^{l} x_{q, \theta(h)_{p}^{-1}(q)} \\
& =\sum_{\sigma_{1}, \ldots, \sigma_{l} \in \mathfrak{S}_{n}} \varphi\left(\theta^{-1}\left(\sigma_{1}, \ldots, \sigma_{l}\right)\right) \prod_{q=1}^{n} \prod_{p=1}^{l} x_{\sigma_{p}(q), q} .
\end{aligned}
$$

We note that $D\left(X ; \alpha^{\nu(\cdot)}\right)=\operatorname{det}^{(\alpha)}(X)^{l}$ since $\nu\left(\theta^{-1}\left(\sigma_{1}, \ldots, \sigma_{l}\right)\right)=\nu\left(\sigma_{1}\right)+\cdots+\nu\left(\sigma_{l}\right)$ for $\left(\sigma_{1}, \ldots, \sigma_{l}\right) \in \mathfrak{S}_{l}^{n}$.

Take a class function $\delta_{H}$ on $H$ defined by

$$
\delta_{H}(h)= \begin{cases}1 & h=1, \\ 0 & h \neq 1 .\end{cases}
$$

We see that $D\left(X ; \delta_{H}\right)=\left(x_{11} x_{22} \ldots x_{n n}\right)^{l}$. We need the following lemma (assertion (1) is just a rewrite of Lemma 2.2, and (2) is easy to verify).

\section{Lemma 6.1.}

(1)

$$
\begin{aligned}
& \mathcal{U}\left(\mathfrak{g l}_{n}\right) \cdot \boldsymbol{e}_{1}^{\otimes l} \otimes \cdots \otimes \boldsymbol{e}_{n}^{\otimes l}=V \cdot e=\mathcal{S}^{l}\left(\mathbb{C}^{n}\right)^{\otimes n} \\
& \mathcal{U}\left(\mathfrak{g l}_{n}\right) \cdot D\left(X ; \delta_{H}\right)=\bigoplus_{\substack{i_{p q} \in\{1,2, \ldots, n\} \\
(1 \leq p \leq l, 1 \leq q \leq n)}} \mathbb{C} \cdot \prod_{q=1}^{n} \prod_{p=1}^{l} x_{i_{p q} q} \cong \mathcal{S}^{l}\left(\mathbb{C}^{n}\right)^{\otimes n}
\end{aligned}
$$

(2) The map

$$
\begin{aligned}
\mathcal{T}: \mathcal{U}\left(\mathfrak{g l}_{n}\right) \cdot D\left(X ; \delta_{H}\right) & \ni \prod_{q=1}^{n} \prod_{p=1}^{l} x_{i_{p q} q} \\
& \longmapsto\left(\boldsymbol{e}_{i_{11}} \otimes \cdots \otimes \boldsymbol{e}_{i_{l 1}}\right) \otimes \cdots \otimes\left(\boldsymbol{e}_{i_{1 n}} \otimes \cdots \otimes \boldsymbol{e}_{i_{l n}}\right) \cdot e \in V \cdot e
\end{aligned}
$$

is a bijective $\mathcal{U}\left(\mathfrak{g l}_{n}\right)$-intertwiner. 
We see that

$$
\begin{aligned}
& \mathcal{T}(D(X ; \varphi)) \\
= & \sum_{h \in H} \varphi(h) \mathcal{T}\left(\prod_{q=1}^{n} \prod_{p=1}^{l} x_{\theta(h)_{p}(q), q}\right) \\
= & \sum_{h \in H} \varphi(h)\left(\boldsymbol{e}_{\theta(h)_{1}(1)} \otimes \cdots \otimes \boldsymbol{e}_{\theta(h)_{l}(1)}\right) \otimes \cdots \otimes\left(\boldsymbol{e}_{\theta(h)_{1}(n)} \otimes \cdots \otimes \boldsymbol{e}_{\theta(h)_{l}(n)}\right) \cdot e \\
= & \boldsymbol{e}_{1}^{\otimes l} \otimes \cdots \otimes \boldsymbol{e}_{n}^{\otimes l} \cdot \sum_{h \in H} \varphi(h) h \cdot e=\boldsymbol{e}_{1}^{\otimes l} \otimes \cdots \otimes \boldsymbol{e}_{n}^{\otimes l} \cdot e \Phi e
\end{aligned}
$$

by (2) in Lemma 6.1. Using (1) in Lemma 6.1, we have

\section{Lemma 6.2.}

$$
\mathcal{U}\left(\mathfrak{g l}_{n}\right) \cdot D(X ; \varphi) \cong V \cdot e \Phi e
$$

as a left $\mathcal{U}\left(\mathfrak{g l}_{n}\right)$-module. In particular, $V \cdot e \Phi e \cong \boldsymbol{V}_{n, l}(\alpha)$ if $\varphi(h)=\alpha^{\nu(h)}$.

By the Schur-Weyl duality, we have

$$
V \cong \bigoplus_{\lambda \vdash n l} \mathcal{M}_{n}^{\lambda} \otimes \mathcal{S}^{\lambda}
$$

Here $\mathcal{S}^{\lambda}$ denotes the irreducible unitary right $\mathfrak{S}_{n l}$-module corresponding to $\lambda$. We see that

$$
\operatorname{dim}\left(\mathcal{S}^{\lambda} \cdot e\right)=\left\langle\operatorname{ind}_{K}^{G} \mathbf{1}_{K}, \mathcal{S}^{\lambda}\right\rangle_{\mathfrak{S}_{n l}}=K_{\lambda\left(l^{n}\right)},
$$

where $\mathbf{1}_{K}$ is the trivial representation of $K$ and $\langle\pi, \rho\rangle_{\mathfrak{S}_{n l}}$ denotes the intertwining number of given representations $\pi$ and $\rho$ of $\mathfrak{S}_{n l}$. Since $K_{\lambda\left(l^{n}\right)}=0$ unless $\ell(\lambda) \leq n$, the next theorem follows.

\section{Theorem 6.3.}

$$
V \cdot e \Phi e \cong \bigoplus_{\substack{\lambda \vdash n l \\ \ell(\lambda) \leq n}} \mathcal{M}_{n}^{\lambda} \otimes\left(\mathcal{S}^{\lambda} \cdot e \Phi e\right) .
$$

In particular, as a left $\mathcal{U}\left(\mathfrak{g l}_{n}\right)$-module, the multiplicity of $\mathcal{M}_{n}^{\lambda}$ in $V \cdot e \Phi e$ is given by

$$
\operatorname{dim}\left(\mathcal{S}^{\lambda} \cdot e \Phi e\right)=\operatorname{rk}_{\operatorname{End}\left(\mathcal{S}^{\lambda} \cdot e\right)}(e \Phi e) .
$$

Let $\lambda \vdash n l$ be a partition such that $\ell(\lambda) \leq n$ and put $d=K_{\lambda\left(l^{n}\right)}$. We fix an orthonormal basis $\left\{\boldsymbol{e}_{1}^{\lambda}, \ldots, \boldsymbol{e}_{f^{\lambda}}^{\lambda}\right\}$ of $\mathcal{S}^{\lambda}$ such that the first $d$ vectors $\boldsymbol{e}_{1}^{\lambda}, \ldots, \boldsymbol{e}_{d}^{\lambda}$ form a subspace $\left(\mathcal{S}^{\lambda}\right)^{K}$ consisting of $K$-invariant vectors and the remaining $f^{\lambda}-d$ vectors form the orthocomplement of $\left(\mathcal{S}^{\lambda}\right)^{K}$ with respect to the $\mathfrak{S}_{n l}$-invariant inner product. The matrix coefficient of $\mathcal{S}^{\lambda}$ relative to this basis is

$$
\psi_{i j}^{\lambda}(g)=\left\langle\boldsymbol{e}_{i}^{\lambda} \cdot g, \boldsymbol{e}_{j}^{\lambda}\right\rangle_{\mathcal{S}^{\lambda}} \quad\left(g \in \mathfrak{S}_{n l}, 1 \leq i, j \leq f^{\lambda}\right) .
$$

We notice that this function is $K$-biinvariant. We see that the multiplicity of $\mathcal{M}_{n}^{\lambda}$ in $V \cdot e \Phi e$ is given by the rank of the matrix

$$
\left(\sum_{h \in H} \varphi(h) \psi_{i j}^{\lambda}(h)\right)_{1 \leq i, j \leq d} .
$$


As a particular case, we obtain

Theorem 6.4. The multiplicity of the irreducible representation $\mathcal{M}_{n}^{\lambda}$ in the cyclic module $\mathcal{U}\left(\mathfrak{g l}_{n}\right) \cdot \operatorname{det}^{(\alpha)}(X)^{l}$ is equal to the rank of

$$
F_{n, l}^{\lambda}(\alpha)=\left(\sum_{h \in H} \alpha^{\nu(h)} \psi_{i j}^{\lambda}(h)\right)_{1 \leq i, j \leq d},
$$

where $\left\{\psi_{i j}^{\lambda}\right\}_{i, j}$ denotes a basis of the $\lambda$-component of the space $C\left(K \backslash \mathfrak{S}_{n l} / K\right)$ of $K$-biinvariant functions on $\mathfrak{S}_{n l}$ given by (6.4).

Remark 6.5.

(1) We have $F_{n, l}^{\lambda}(0)=I$ by the definition of the basis $\left\{\psi_{i j}^{\lambda}\right\}_{i, j}$ in (6.4).

(2) Since $\alpha^{\nu\left(g^{-1}\right)}=\alpha^{\nu(g)}$ and $\psi_{i j}^{\lambda}\left(g^{-1}\right)=\overline{\psi_{j i}^{\lambda}(g)}$ for any $g \in \mathfrak{S}_{n l}$, the transition matrices satisfy $F_{n, l}^{\lambda}(\alpha)^{*}=F_{n, l}^{\lambda}(\bar{\alpha})$.

(3) In Examples 6.6 and 6.8 below, the transition matrices are given by diagonal matrices. We expect that any transition matrix $F_{n, l}^{\lambda}(\alpha)$ is diagonalizable in $\operatorname{Mat}_{K_{\lambda\left(l^{n}\right)}}(\mathbb{C}[\alpha])$.

Example 6.6. If $l=1$, then $H=G=\mathfrak{S}_{n}$ and $K=\{1\}$. Therefore, for any $\lambda \vdash n$, we have

$$
F_{n, 1}^{\lambda}(\varphi)=\frac{n !}{f^{\lambda}}\left\langle\varphi, \chi^{\lambda}\right\rangle_{\mathfrak{S}_{n}} I
$$

by the orthogonality of the matrix coefficients. Here $\chi^{\lambda}$ denotes the irreducible character of $\mathfrak{S}_{n}$ corresponding to $\lambda$. In particular, if $\varphi=\alpha^{\nu(\cdot)}$, then

$$
F_{n, 1}^{\lambda}(\alpha)=f_{\lambda}(\alpha) I
$$

since the Fourier expansion of $\alpha^{\nu(\cdot)}$ (as a class function on $\mathfrak{S}_{n}$ ) is

$$
\alpha^{\nu(\cdot)}=\sum_{\lambda \vdash n} \frac{f^{\lambda}}{n !} f_{\lambda}(\alpha) \chi^{\lambda},
$$

which is obtained by specializing the Frobenius character formula for $\mathfrak{S}_{n}$ (see e.g. [10]).

Example 6.7. Let us calculate $F_{n, l}^{(n l)}(\alpha)$ by using Theorem 6.4, Since $\mathcal{S}^{(n l)}$ is the trivial representation, it follows that $\left(\mathcal{S}^{(n l)}\right)^{K}=\mathcal{S}^{(n l)}$ and

$$
\begin{aligned}
F_{n, l}^{(n l)}(\alpha)=\sum_{h \in H} \alpha^{\nu(h)}\langle\boldsymbol{e} \cdot h, \boldsymbol{e}\rangle=\sum_{\sigma_{1}, \ldots, \sigma_{l} \in \mathfrak{S}_{n}} \alpha^{\nu\left(\sigma_{1}\right)} \ldots \alpha^{\nu\left(\sigma_{l}\right)} & \\
& =((1+\alpha)(1+2 \alpha) \ldots(1+(n-1) \alpha))^{l},
\end{aligned}
$$

where $\boldsymbol{e}$ denotes a unit vector in $\mathcal{S}^{(n l)}$.

Example 6.8. Let us calculate $F_{n, l}^{(n l-1,1)}(\alpha)$ by using Theorem 6.4 As is well known, the irreducible (right) $\mathfrak{S}_{n l}$-module $\mathcal{S}^{(n l-1,1)}$ can be realized in $\mathbb{C}^{n l}$ as follows:

$$
\mathcal{S}^{(n l-1,1)}=\left\{\left(x_{j}\right)_{j=1}^{n l} \in \mathbb{C}^{n l} \mid \sum_{j=1}^{n l} x_{j}=0\right\} .
$$


This is a unitary representation with respect to the ordinary hermitian inner product $\langle\cdot, \cdot\rangle$ on $\mathbb{C}^{n l}$. We can immediately see that

$$
\begin{aligned}
& \left(\mathcal{S}^{(n l-1,1)}\right)^{K} \\
& \quad=\left\{\left(x_{j}\right)_{j=1}^{n l} \in \mathcal{S}^{(n l-1,1)} \mid x_{p l+1}=x_{p l+2}=\cdots=x_{(p+1) l} \quad(0 \leq p<n)\right\} .
\end{aligned}
$$

Take an orthonormal basis $\boldsymbol{e}_{1}, \ldots, \boldsymbol{e}_{n-1}$ of $\left(\mathcal{S}^{(n l-1,1)}\right)^{K}$ by letting

$$
\boldsymbol{e}_{j}=\frac{1}{\sqrt{n l}}(\overbrace{\omega^{j}, \ldots, \omega^{j}}^{l}, \overbrace{\omega^{2 j}, \ldots, \omega^{2 j}}^{l}, \ldots, \overbrace{\omega^{n j}, \ldots, \omega^{n j}}^{l}) \quad(1 \leq j \leq n-1),
$$

where $\omega$ is a primitive $n$-th root of unity. Then, the $(i, j)$-entry of the transition matrix $F_{n, l}^{(n l-1,1)}(\alpha)$ is

$$
\begin{aligned}
\sum_{h \in H} \alpha^{\nu(h)}\left\langle\boldsymbol{e}_{i} \cdot h, \boldsymbol{e}_{j}\right\rangle & =\frac{1}{n l} \sum_{\sigma_{1}, \ldots, \sigma_{l} \in \mathfrak{S}_{n}} \sum_{p=1}^{n} \sum_{q=1}^{l} \alpha^{\nu\left(\sigma_{1}\right)} \ldots \alpha^{\nu\left(\sigma_{l}\right)} \omega^{\sigma_{q}(p) i-p j} \\
& =\left(\sum_{\tau \in \mathfrak{S}_{n}} \alpha^{\nu(\tau)}\right)^{l-1}\left(\frac{1}{n} \sum_{\sigma \in \mathfrak{S}_{n}} \sum_{p=1}^{n} \alpha^{\nu(\sigma)} \omega^{\sigma(p) i-p j}\right) .
\end{aligned}
$$

The first factor is $((1+\alpha)(1+2 \alpha) \ldots(1+(n-1) \alpha))^{l-1}$. We shall show that

$$
\begin{array}{r}
\frac{1}{n} \sum_{\sigma \in \mathfrak{S}_{n}} \sum_{p=1}^{n} \alpha^{\nu(\sigma)} \omega^{\sigma(p) i-p j}=(1-\alpha)(1+\alpha)(1+2 \alpha) \ldots(1+(n-2) \alpha) \delta_{i j} \\
(i, j=1,2, \ldots, n-1) .
\end{array}
$$

For this purpose, by comparing the coefficients of $\alpha^{n-m}$ in both sides, it is enough to prove that

$$
\frac{1}{n} \sum_{\substack{\sigma \in \mathfrak{S}_{n} \\
\nu(\sigma)=n-m}} \sum_{p=1}^{n} \omega^{\sigma(p) i-p j}=\left\{\left[\begin{array}{c}
n-1 \\
m-1
\end{array}\right]-\left[\begin{array}{c}
n-1 \\
m
\end{array}\right]\right\} \delta_{i j}
$$

$$
(i, j, m=1,2, \ldots, n-1),
$$

where $\left[\begin{array}{c}n \\ m\end{array}\right]$ denotes the Stirling number of the first kind (see e.g. [6] for the definition). Since

$$
\left|\left\{\sigma \in \mathfrak{S}_{n} ; \nu(\sigma)=n-m, \sigma(p)=x\right\}\right|=\left\{\begin{array}{cc}
{\left[\begin{array}{c}
n-1 \\
m-1
\end{array}\right]} & x=p \\
{\left[\begin{array}{c}
n-1 \\
m
\end{array}\right]} & x \neq p
\end{array}\right.
$$

for each $p, x \in[n]$, it follows that

$$
\begin{aligned}
\frac{1}{n} \sum_{\sigma \in \mathfrak{S}_{n}} \sum_{p=1}^{n} \alpha^{\nu(\sigma)} \omega^{\sigma(p) i-p j}=\frac{1}{n} \sum_{p=1}^{n} \omega^{-p j}\left\{\left[\begin{array}{c}
n-1 \\
m-1
\end{array}\right] \omega^{p i}+\sum_{x \neq p}\left[\begin{array}{c}
n-1 \\
m
\end{array}\right] \omega^{x i}\right\} \\
=\left\{\left[\begin{array}{c}
n-1 \\
m-1
\end{array}\right]-\left[\begin{array}{c}
n-1 \\
m
\end{array}\right]\right\} \frac{1}{n} \sum_{p=1}^{n} \omega^{p(i-j)}=\left\{\left[\begin{array}{c}
n-1 \\
m-1
\end{array}\right]-\left[\begin{array}{c}
n-1 \\
m
\end{array}\right]\right\} \delta_{i j},
\end{aligned}
$$


which is the required conclusion. Here we notice that $\sum_{x \neq p} \omega^{x i}=-\omega^{p i}$ since $1 \leq i<n$. Consequently, we obtain

$$
\begin{aligned}
& F_{n, l}^{(n l-1,1)}(\alpha) \\
= & \left((1-\alpha)((1+\alpha)(1+2 \alpha) \ldots(1+(n-2) \alpha))^{l}(1+(n-1) \alpha)^{l-1} \delta_{i j}\right)_{1 \leq i, j \leq n-1},
\end{aligned}
$$

so that the multiplicity of $\mathcal{M}_{n}^{(n l-1,1)}$ in $\boldsymbol{V}_{n, l}(\alpha)$ is zero if $\alpha=-1 / k(k=1,2, \ldots, n-$ 1) and $n-1$ otherwise.

The trace of the transition matrix $F_{n, l}^{\lambda}(\alpha)$ is

$$
f_{n, l}^{\lambda}(\alpha)=\operatorname{tr} F_{n, l}^{\lambda}(\alpha)=\sum_{h \in H} \alpha^{\nu(h)} \omega^{\lambda}(h)
$$

where $\omega^{\lambda}$ is the zonal spherical function for $\lambda$ with respect to $K$, defined by

$$
\omega^{\lambda}(g)=\frac{1}{|K|} \sum_{k \in K} \chi^{\lambda}(k g) \quad\left(g \in \mathfrak{S}_{n l}\right) .
$$

This polynomial is regarded as a generalization of the modified content polynomial since $f_{n, 1}^{\lambda}(\alpha)=f^{\lambda} f_{\lambda}(\alpha)$ as we have seen above. It is much easier to handle these polynomials than the transition matrices. If we could prove that a transition matrix $F_{n, l}^{\lambda}(\alpha)$ is a scalar matrix, then we would have $F_{n, l}^{\lambda}(\alpha)=d^{-1} f_{n, l}^{\lambda}(\alpha) I$ $\left(d=\operatorname{dim}\left(\mathcal{S}^{\lambda}\right)^{K}\right)$ and hence we see that the multiplicity of $\mathcal{M}_{n}^{\lambda}$ in $\boldsymbol{V}_{n, l}(\alpha)$ is completely controlled by the single polynomial $f_{n, l}^{\lambda}(\alpha)$. In this sense, it is desirable to obtain a characterization of the irreducible representations whose corresponding transition matrices are scalar as well as to get an explicit expression for the polynomials $f_{n, l}^{\lambda}(\alpha)$. Here we give a sufficient condition on $\lambda \vdash n l$ for $F_{n, l}^{\lambda}(\alpha)$ to be a scalar matrix.

Proposition 6.9. (1) Denote by $N_{H}(K)$ the normalizer of $K$ in $H$. The transition matrix $F_{n, l}^{\lambda}(\alpha)$ is scalar if $\left(\mathcal{S}^{\lambda}\right)^{K}$ is irreducible as a $N_{H}(K)$-module.

(2) If $\lambda$ is of hook-type (i.e. $\lambda=\left(n l-r, 1^{r}\right)$ for some $\left.r<n\right)$, then $F_{n, l}^{\lambda}(\alpha)$ is scalar.

Proof. Notice that $N_{H}(K) \cong \mathfrak{S}_{n}$. Consider a linear map $T \in \operatorname{End}\left(\left(\mathcal{S}^{\lambda}\right)^{K}\right)$ given by

$$
T(\boldsymbol{x})=\sum_{j=1}^{d}\left(\sum_{h \in H} \alpha^{\nu(h)}\left\langle\boldsymbol{x} \cdot h, \boldsymbol{e}_{j}^{\lambda}\right\rangle_{\mathcal{S}^{\lambda}}\right) \boldsymbol{e}_{j}^{\lambda} \quad\left(\boldsymbol{x} \in\left(\mathcal{S}^{\lambda}\right)^{K}\right),
$$

where $d=\operatorname{dim}\left(\mathcal{S}^{\lambda}\right)^{K}$. It is straightforward to check that $T$ gives an intertwiner of $\left(\mathcal{S}^{\lambda}\right)^{K}$ as a $N_{H}(K)$-module. Hence, by Schur's lemma, $T$ is a scalar map (and $F_{n, l}^{\lambda}(\alpha)$ is a scalar matrix) if $\left(\mathcal{S}^{\lambda}\right)^{K}$ is an irreducible $N_{H}(K)$-module. When $\lambda=$ $\left(n l-r, 1^{r}\right)$ for some $r<n$, it is proved in [2, Proposition 5.3] that $\left(\mathcal{S}^{\left(n l-r, 1^{r}\right)}\right)^{K} \cong$ $\mathcal{S}^{\left(n-r, 1^{r}\right)}$ as $N_{H}(K)$-modules. Thus we have the proposition. 
Example 6.10. Let us calculate $f_{n, l}^{(n l-1,1)}(\alpha)$. Notice that $\chi^{(n l-1,1)}(g)=\mathrm{fix}_{n l}(g)-1$ where fix $_{n l}$ denotes the number of fixed points in the natural action $\mathfrak{S}_{n l} \curvearrowright[n l]$. Hence we see that

$$
\begin{aligned}
f_{n, l}^{(n l-1,1)}(\alpha)=\sum_{h \in H} \alpha^{\nu(h)} \frac{1}{|K|} \sum_{k \in K}\left(\operatorname{fix}_{n l}(k h)-1\right) \\
\quad=\sum_{h \in H} \alpha^{\nu(h)} \frac{1}{|K|} \sum_{k \in K} \sum_{x \in[n l]} \delta_{k h x, x}-\sum_{h \in H} \alpha^{\nu(h)} .
\end{aligned}
$$

It is easily seen that $k h x \neq x$ for any $k \in K$ if $h x \neq x(x \in[n l])$. Thus it follows that

$$
\frac{1}{|K|} \sum_{k \in K} \sum_{x \in[n l]} \delta_{k h x, x}=\sum_{x \in[n l]} \delta_{h x, x} \frac{1}{|K|} \sum_{k \in K} \delta_{k x, x}=\frac{1}{l} \mathrm{fix}_{n l}(h) \quad(h \in H) .
$$

Therefore we have

$$
\begin{aligned}
f_{n, l}^{(n l-1,1)}(\alpha) & =\frac{1}{l} \sum_{h \in H} \alpha^{\nu(h)} \operatorname{fix}_{n l}(h)-\sum_{h \in H} \alpha^{\nu(h)}=f_{n, 1}^{(n)}(\alpha)^{l-1} f_{n, 1}^{(n-1,1)}(\alpha) \\
& =(n-1)(1-\alpha)(1-(n-1) \alpha)^{l-1} \prod_{i=1}^{n-2}(1+i \alpha)^{l} .
\end{aligned}
$$

Since the transition matrix $F_{n, l}^{(n l-1,1)}$ is scalar and its size is $\operatorname{dim} \mathcal{S}^{(n-1,1)}=n-1$, we get $F_{n, l}^{(n l-1,1)}(\alpha)=(1-\alpha)(1-(n-1) \alpha)^{l-1} \prod_{i=1}^{n-2}(1+i \alpha)^{l} I_{n-1}$ again.

We will investigate these polynomials $f_{n, l}^{\lambda}(\alpha)$ and their generalizations in [7].

6.2. Irreducible decomposition of $\boldsymbol{V}_{2, l}(\alpha)$ and Jacobi polynomials. In this subsection, as a particular example, we consider the case where $n=2$ and calculate the transition matrix $F_{2, l}^{\lambda}(\alpha)$ explicitly. Since the pair $\left(\mathfrak{S}_{2 l}, K\right)$ is a Gelfand pair (see e.g. [10]), it follows that

$$
K_{\lambda\left(l^{2}\right)}=\left\langle\operatorname{ind}_{K}^{\mathfrak{S}_{2 l}} \mathbf{1}_{K}, \mathcal{S}^{\lambda}\right\rangle_{\mathfrak{S}_{2 l}}=1
$$

for each $\lambda \vdash 2 n$ with $\ell(\lambda) \leq 2$. Thus, in this case, the transition matrix is just a polynomial and is given by

$$
F_{2, l}^{\lambda}(\alpha)=\operatorname{tr} F_{2, l}^{\lambda}(\alpha)=\sum_{h \in H} \alpha^{\nu(h)} \omega^{\lambda}(h)=\sum_{s=0}^{l}\left(\begin{array}{l}
l \\
s
\end{array}\right) \omega^{\lambda}\left(g_{s}\right) \alpha^{s}
$$

Here we put $g_{s}=(1, l+1)(2, l+2) \ldots(s, l+s) \in \mathfrak{S}_{2 n}$. Now we write $\lambda=(2 l-p, p)$ for some $p(0 \leq p \leq l)$. The value $\omega^{(2 l-p, p)}\left(g_{s}\right)$ of the zonal spherical function is calculated by Bannai and Ito [3, p. 218] as

$$
\omega^{(2 l-p, p)}\left(g_{s}\right)=Q_{p}(s ;-l-1,-l-1, l)=\sum_{j=0}^{p}(-1)^{j}\left(\begin{array}{c}
p \\
j
\end{array}\right)\left(\begin{array}{c}
2 l-p+1 \\
j
\end{array}\right)\left(\begin{array}{l}
l \\
j
\end{array}\right)^{-2}\left(\begin{array}{l}
s \\
j
\end{array}\right),
$$


where

$$
\begin{aligned}
Q_{n}(x ; \alpha, \beta, N) & ={ }_{3} \tilde{F}_{2}\left(\begin{array}{c}
-n, n+\alpha+\beta+1,-x \\
\alpha+1,-N
\end{array}\right) \\
& =\sum_{j=0}^{N}(-1)^{j}\left(\begin{array}{c}
n \\
j
\end{array}\right)\left(\begin{array}{c}
-n-\alpha-\beta-1 \\
j
\end{array}\right)\left(\begin{array}{c}
-\alpha-1 \\
j
\end{array}\right)^{-1}\left(\begin{array}{c}
N \\
j
\end{array}\right)^{-1}\left(\begin{array}{l}
x \\
j
\end{array}\right)
\end{aligned}
$$

is the Hahn polynomial (see also [10, p. 399]), and ${ }_{n+1} \tilde{F}_{n}\left(\begin{array}{c}a_{1}, \ldots, a_{p} \\ b_{1}, \ldots, b_{q-1},-N\end{array} ; x\right)$ is the hypergeometric polynomial

$$
{ }_{p} \tilde{F}_{q}\left(\begin{array}{c}
a_{1}, \ldots, a_{p} \\
b_{1}, \ldots, b_{q-1},-N
\end{array} ; x\right)=\sum_{j=0}^{N} \frac{\left(a_{1}\right)_{j} \ldots\left(a_{p}\right)_{j}}{\left(b_{1}\right)_{j} \ldots\left(b_{q-1}\right)_{j}(-N)_{j}} \frac{x^{j}}{j !}
$$

for $p, q, N \in \mathbb{N}$ in general (see [1]). We now re-state Theorem 4.1 as follows:

Theorem 6.11. Let $l$ be a positive integer. Then

$$
F_{2, l}^{(2 l-p, p)}(\alpha)=\sum_{s=0}^{l}\left(\begin{array}{l}
l \\
s
\end{array}\right) Q_{p}(s ; l-1, l-1, l) \alpha^{s}=(1+\alpha)^{l-p} G_{p}^{l}(\alpha)
$$

for $p=0,1, \ldots, l$.

Proof. Let us put $x=-1 / \alpha$. Then we have

$$
\begin{aligned}
& \sum_{s=0}^{l}\left(\begin{array}{l}
l \\
s
\end{array}\right) Q_{p}(s ; l-1, l-1, l) \alpha^{s} \\
= & \sum_{j=0}^{p}(-1)^{j}\left(\begin{array}{l}
p \\
j
\end{array}\right)\left(\begin{array}{c}
2 l-p+1 \\
j
\end{array}\right)\left(\begin{array}{l}
l \\
j
\end{array}\right)^{-1} \alpha^{j}(1+\alpha)^{l-j} \\
= & x^{-l}(x-1)^{l-p} \sum_{j=0}^{p}\left(\begin{array}{c}
p \\
j
\end{array}\right)\left(\begin{array}{c}
2 l-p+1 \\
j
\end{array}\right)\left(\begin{array}{l}
l \\
j
\end{array}\right)^{-1}(x-1)^{p-j}
\end{aligned}
$$

and

$$
(1+\alpha)^{l-p} G_{p}^{l}(\alpha)=x^{-l}(x-1)^{l-p} \sum_{j=0}^{p}(-1)^{j}\left(\begin{array}{c}
p \\
j
\end{array}\right)\left(\begin{array}{c}
l-p+j \\
j
\end{array}\right)\left(\begin{array}{l}
l \\
j
\end{array}\right)^{-1}(-x)^{p-j} .
$$

Here we use the elementary identity

$$
\sum_{s=0}^{l}\left(\begin{array}{l}
l \\
s
\end{array}\right)\left(\begin{array}{l}
s \\
j
\end{array}\right) \alpha^{s}=\left(\begin{array}{l}
l \\
j
\end{array}\right) \alpha^{j}(1+\alpha)^{l-j}
$$

Hence, to prove the theorem, it is enough to verify

$$
\sum_{i=0}^{p}\left(\begin{array}{c}
p \\
i
\end{array}\right)\left(\begin{array}{c}
l-p+i \\
i
\end{array}\right)\left(\begin{array}{l}
l \\
i
\end{array}\right)^{-1} x^{p-i}=\sum_{j=0}^{p}\left(\begin{array}{l}
p \\
j
\end{array}\right)\left(\begin{array}{c}
2 l-p+1 \\
j
\end{array}\right)\left(\begin{array}{l}
l \\
j
\end{array}\right)^{-1}(x-1)^{p-j}
$$

Comparing the coefficients of the Taylor expansions of these polynomials at $x=1$, we notice that the proof is reduced to showing the equality

$$
\sum_{i=0}^{r}\left(\begin{array}{l}
l-i \\
l-r
\end{array}\right)\left(\begin{array}{c}
l-p+i \\
l-p
\end{array}\right)=\left(\begin{array}{c}
2 l-p+1 \\
r
\end{array}\right)
$$


for $0 \leq r \leq p$, which is well known (see e.g. (5.26) in [6]). Hence we have the conclusion.

Thus we obtain the irreducible decomposition

$$
\boldsymbol{V}_{2, l}(-1) \cong \mathcal{M}_{2}^{(l, l)}, \quad \boldsymbol{V}_{2, l}(\alpha) \cong \bigoplus_{\substack{0 \leq p \leq l \\ G_{p}^{l}(\alpha) \neq 0}} \mathcal{M}_{2}^{(2 l-p, p)} \quad(\alpha \neq-1)
$$

of $\boldsymbol{V}_{2, l}(\alpha)$ again.

Remark 6.12 .

(1) The calculation above takes advantage of the fact that the pair $\left(\mathfrak{S}_{n l}, \mathfrak{S}_{l}^{n}\right)$ is the Gelfand pair only when $n=2$.

(2) We have used the result in [3, p. 218] to obtain the theorem. It is worth mentioning that, conversely, one may prove the result in [3, p. 218] from Theorem 4.1

\section{ACKNOWLEDGMENT}

The first author would like to thank Professor Itaru Terada for pointing out that his work 2 is useful for the discussion in Section 6.2. The authors would also like to thank Jyoichi Kaneko for fruitful discussions on Jacobi polynomials.

\section{REFERENCES}

1. G. E. Andrews, R. Askey and R. Roy, Special Functions, Encyclopedia of Mathematics and its Applications 71, Cambridge University Press, Cambridge, 1999. MR.1688958 (2000g:33001)

2. S. Ariki, J. Matsuzawa and I. Terada, Representation of Weyl groups on zero weight spaces of $\mathfrak{g}$-modules, Algebraic and Topological Theories (Kinosaki, 1984), 546-568, Kinokuniya, Tokyo, 1986. MR 1102274

3. E. Bannai and T. Ito, Algebraic Combinatorics I, Association Schemes, The Benjamin/Cummings Publishing Co., Inc., Menlo Park, CA, 1984. MR0882540 (87m:05001)

4. W. Fulton and J. Harris, Representation theory. A first course, Graduate Texts in Mathematics 129, Readings in Mathematics, Springer-Verlag, New York, 1991. MR.1153249 (93a:20069)

5. I. M. Gel'fand and M. A. Naŭmark, Unitary representations of the Lorentz group, Acad. Sci. USSR J. Phys. 10 (1946), 93-94; Izvestiya Akad. Nauk SSSR Ser. Mat. 11 (1947), 411-504. MR.0017282 (8:132b)

6. R. L. Graham, D. E. Knuth and O. Patashnik, Concrete Mathematics. A foundation for computer science, Second edition, Addison-Wesley Publishing Company, Reading, MA, 1994. MR.1397498 (97d:68003)

7. K. Kimoto, Generalized content polynomials toward $\alpha$-determinant cyclic modules, Preprint (2007).

8. K. Kimoto and M. Wakayama, Invariant theory for singular $\alpha$-determinants, J. Combin. Theory Ser. A 115 (2008), no. 1, 1-31. MR2378855

9. $\longrightarrow$ Quantum $\alpha$-determinant cyclic modules of $\mathcal{U}_{q}\left(\mathfrak{g l}_{n}\right)$, J. Algebra 313 (2007), 922-956. MR2329577 (2008d:17021)

10. I. G. Macdonald, Symmetric Functions and Hall Polynomials, 2nd edn., Oxford University Press, 1995. MR1354144 (96h:05207)

11. S. Matsumoto, Alpha-pfaffian, pfaffian point process and shifted Schur measure, Linear Algebra Appl. 403 (2005), 369-398. MR 2140292 (2006d:15014)

12. S. Matsumoto and M. Wakayama, Alpha-determinant cyclic modules of $\mathfrak{g l}_{n}(\mathbb{C})$, J. Lie Theory 16 (2006), 393-405. MR2197599 (2007a:17011)

13. T. Shirai and Y. Takahashi, Random point fields associated with certain Fredholm determinants I: fermion, Poisson and boson point processes, J. Funct. Anal. 205 (2003), 414-463. MR 2018415 (2004m:60104)

14. S. Yu. Slavyanov and W. Lay, Special Functions - A Unified Theory Based on Singularities, Oxford Univ. Press, Oxford, 2000. MR,1858237 (2004e:33003) 
15. G. Szegö, Orthogonal Polynomials, 4th edn., American Mathematical Society, 1975. MR 0372517 (51:8724)

16. D. Vere-Jones, A generalization of permanents and determinants, Linear Algebra Appl. 111 (1988), 119-124. MR0974048 (89j:15014)

17. H. Weyl, The Classical Groups: Their Invariants and Representations, Fifteenth printing, Princeton Landmarks in Mathematics, Princeton University Press, 1997. MR 1488158 (98k:01049)

Department of Mathematical Sciences, University of the Ryukyus, Nishihara, OkiNAWA 903-0213, JAPAN

E-mail address: kimoto@math.u-ryukyu.ac.jp

FaCulty of Mathematics, Kyushu University, Hakozaki Higashi-Ku, FukUOKa 812-8581, JAPAN

Current address: Department of Mathematics, Nagoya University, Chikusa, Nagoya 464-8602, Japan

E-mail address: sho-matsumoto@math.nagoya-u.ac.jp

FaCulty of Mathematics, Kyushu University, HakOZaki Higashi-Ku, FukUOKa 812-8581, JAPAN

E-mail address: wakayama@math.kyushu-u.ac.jp 